\title{
Synthesis of 3-substituted 1-phenyl-1H-pyrazole-4-carbaldehydes and the corresponding ethanones by Pd-catalysed cross-coupling reactions
}

\author{
Eglė Arbačiauskienė, ${ }^{a}$ Vytas Martynaitis, ${ }^{\text {b }}$ Sonata Krikštolaitytė, ${ }^{b}$ Wolfgang Holzer, ${ }^{\text {* } *}$ and \\ Algirdas Šačkus ${ }^{\mathrm{a} *}$ \\ ${ }^{a}$ Institute of Synthetic Chemistry, Kaunas University of Technology, Radvilenu pl. 19, LT-50254 \\ Kaunas, Lithuania \\ ${ }^{b}$ Department of Organic Chemistry, Faculty of Chemical Technology, Kaunas University of \\ Technology, Radvilenu pl. 19, LT-50254 Kaunas, Lithuania \\ ${ }^{c}$ Department of Drug and Natural Product Synthesis, Faculty of Life Sciences, University of \\ Vienna, Althanstrasse 14, A-1090 Vienna, Austria \\ E-mail: algirdas.sackus@ktu.lt,wolfgang.holzer@univie.at
}

\begin{abstract}
An efficient synthetic route to construct ortho-substituted 1-phenyl-1H-pyrazole-4carboxaldehydes and the corresponding ethanones starting from 1-phenyl-1H-pyrazol-3-ol is described. Carbon-carbon bond-forming Pd-catalysed cross-coupling reactions were applied for the functionalisation of the intermediate pyrazole triflates. Detailed NMR spectroscopic investigations were undertaken with all obtained products.
\end{abstract}

Keywords: Pyrazole, triflate, Pd-catalysed reactions, NMR $\left({ }^{1} \mathrm{H},{ }^{13} \mathrm{C},{ }^{15} \mathrm{~N},{ }^{19} \mathrm{~F}\right)$

\section{Introduction}

Palladium-catalysed cross-coupling reactions have proven to be one of the most powerful tools for the formation of the carbon-carbon bond in organic synthesis due to their excellent selectivity and broad tolerance for functional groups. ${ }^{1}$ In recent years, this method has been increasingly employed for the functionalisation of nitrogen heterocycles. ${ }^{2}$ A number of accordant studies on azoles with these reactions have been published. ${ }^{3}$ These heteroarenes represent the prevalent substructures for a variety of target compounds, with applications ranging from pharmaceuticals to molecular organic materials. In particular, substituted pyrazoles (1,2-diazoles) are interesting building blocks and frequently encountered structural units in a variety of pharmaceuticals, ${ }^{4}$ agrochemicals, ${ }^{5}$ dyes ${ }^{6}$ and complexing agents. ${ }^{7}$ Appropriate pyrazole halides have mainly been used as precursors for the functionalisation of pyrazoles via Heck, ${ }^{8}$ Suzuki, ${ }^{8,9}$ Sonogashira, ${ }^{8,9 d-f, 10}$ 
and Stille couplings ${ }^{8,9 e, 11}$. Recently, pyrazole triflates have also been employed in Suzuki ${ }^{12}$ and Negishi couplings. ${ }^{13}$ These reactive species are readily available by triflation of the corresponding pyrazolones (hydroxypyrazoles), which are frequently available from commercial sources. The use of $O$-triflated pyrazoles as common intermediates for the preparation of orthosubstituted pyrazoles via palladium-mediated cross-coupling strategies remains largely unexplored but is anticipated as a powerful synthetic approach for the functionalisation of such compounds, especially to obtain fused systems containing pyrazole subunits.

In this respect, (hetero)aromatic systems bearing an alkyne moiety and a formyl (or acetyl) group in ortho-position represent suitable starting materials for various annulation reactions leading to the formation of condensed heterocyclic systems. Recent examples for such a synthetic approach include the transformations of 2-alkynylquinoline-3-carbaldehydes into benzo[b][1,6]naphthyridines, ${ }^{14}$ 4-alkynylpyrimidine-5-carbaldehydes into quinozolines or pyrido[4,3-d]pyrimidines, ${ }^{15}$ and 5-acetyl-4-alkynylthiazoles into the corresponding pyrido[3,4c] thiazoles. ${ }^{16}$

Moreover, an acetyl group and a phenyl ring attached at the ortho-position on a (hetero)aromatic system can be successfully employed for annulation reactions. For example, 2acetylbiphenyl, obtained by the Suzuki reaction from ortho-bromoacetophenone, was easily transformed into phenanthridine via a dioxime oxalate intermediate. ${ }^{17}$

Given this proven utility, it seems reasonable that the development of efficient synthetic methods for the construction of new ortho-substituted heterocycles from easily available starting materials might provide additional lead molecules for use in drug discovery and for the development of advanced materials. Thus, we herein report on our corresponding adaption of the above approach to ortho-substituted pyrazoles including (1) the efficient synthesis of the starting 3-triflyloxy-1-phenyl-1H-pyrazole-4-carbaldehyde and the corresponding ethanone from the common synthetic precursor 1-phenyl-1H-pyrazol-3-ol, and (2) the preparation of the target 3substituted products by the palladium mediated cross-coupling reactions of the intermediate triflates with (hetero)aryl boronic acids, phenylacetylene and various alkenes.

\section{Results and Discussion}

Triflates 7 and 9 represent the starting materials for our coupling reactions and hence are the key compounds of our investigations. Their synthesis was accomplished according to Scheme 1, originating from 3-hydroxy-1-phenyl-1H-pyrazole $\mathbf{1}$ as a common starting material. The latter was obtained from commercially available 1-phenylpyrazolidin-3-one following a known procedure. $^{18}$

3-Hydroxy-1-phenyl-1H-pyrazole-4-carbaldehyde 6, the precursor for the preparation of the triflate 7 , was obtained by a series of reactions including formylation at the 4-position. Although the preparation of $\mathbf{6}$ by oxidation of (3-benzyloxy-1-phenyl-1H-pyrazol-4-yl)methanol with 
manganese dioxide and subsequent catalytic debenzylation is reported in a patent application, neither a source of the starting material nor spectroscopic data of the products were provided. ${ }^{19}$

Several methods are known for the formylation of pyrazoles at the 4-position of the heteroaromatic ring, comprising synthesis via Grignard intermediates, ${ }^{20}$ bromine-lithium exchange or direct ortho-lithiation followed by treatment with $\mathrm{DMF},{ }^{21}$ and the Vilsmeier-Haack reaction. ${ }^{22}$ However, the-above mentioned metallation methods are not usually suitable in the case of unprotected hydroxyl derivatives, while formylation of hydroxypyrazoles by treatment with $\mathrm{DMF} / \mathrm{POCl}_{3}$ can lead to the replacement of the hydroxyl group by a chlorine atom. ${ }^{23}$

Facing these challenges, we explored two alternative synthetic strategies in order to obtain the desired aldehyde 6 (Scheme 1). The first approach is based on the transformation of compound $\mathbf{1}$ into a species suitable for selective lithiation. To this purpose, $\mathbf{1}$ was brominated according to a known procedure. ${ }^{18}$ The obtained product 2 was further benzylated with benzyl chloride under standard conditions in alkaline medium to afford $O$-benzyl-4-bromo-1-phenyl$1 H$-pyrazole 3. Treatment of 3 with $n$-BuLi at $-78{ }^{\circ} \mathrm{C}$ gave rise to selective bromine-lithium exchange. Subsequent quenching of the intermediate 4-lithiopyrazole with 1.5 equiv of DMF afforded carbaldehyde 4 in $70 \%$ yield.

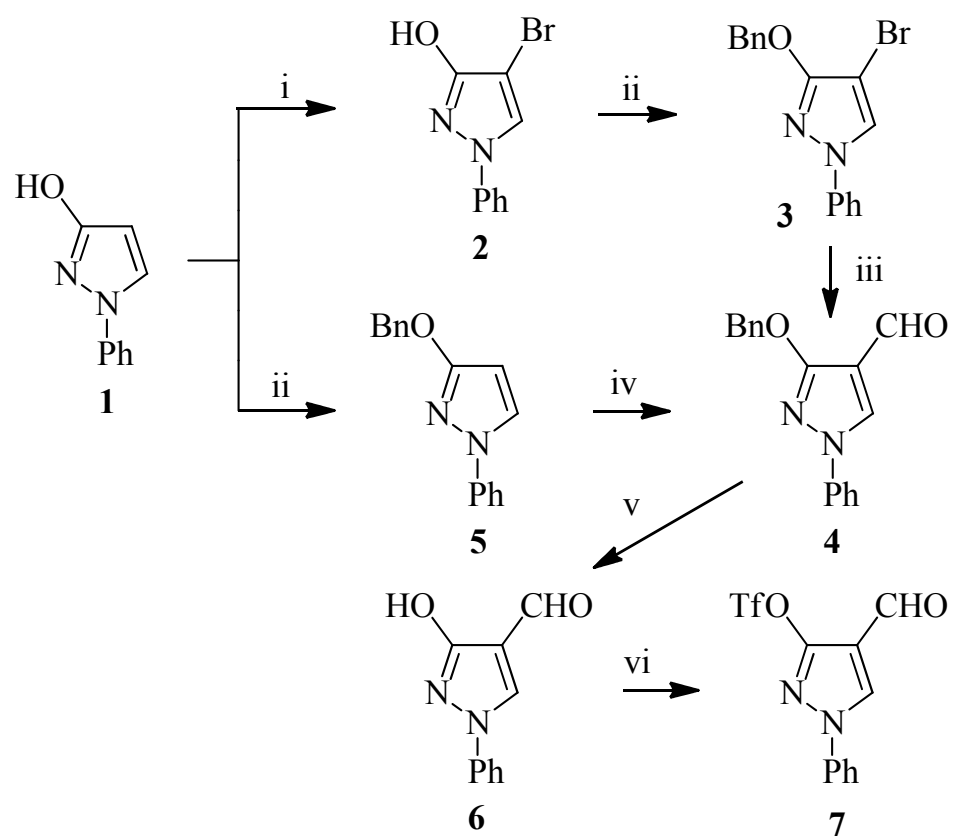

Scheme 1. Reagents and conditions: i: $\mathrm{Br}_{2}, \mathrm{CHCl}_{3}$, rt, 20 h, 75\%; ii: $\mathrm{BnCl}, \mathrm{NaH}, \mathrm{DMF}, 0-60{ }^{\circ} \mathrm{C}$, $1 \mathrm{~h}, 73 \%$ for 3, $85 \%$ for 5; iii: $n$-BuLi, THF, DMF, $-78{ }^{\circ} \mathrm{C}, \mathrm{rt}, 0.5 \mathrm{~h}, 70 \%$; iv: $\mathrm{POCl}_{3}, \mathrm{DMF}, 70$ ${ }^{\circ} \mathrm{C}$, overnight, $60 \%$; v: TFA, rt, 2 days, $85 \%$; vi: Tf 2 O, TEA, DCM, rt, 1 h, $83 \%$.

To find a more convenient method for the preparation of aldehyde 4, we next explored the Vilsmeier-Haack reaction of 4-benzyloxy-1-phenyl-1H-pyrazole 5, which was obtained by 
benzylation of the starting compound $\mathbf{1}$. After heating compound 5 with $\mathrm{DMF} / \mathrm{POCl}_{3}$ at $70{ }^{\circ} \mathrm{C}$ for $12 \mathrm{~h}$, the reaction gave the target carbaldehyde 4 in $60 \%$ yield.

Debenzylation of compound $\mathbf{4}$ was accomplished by treatment with TFA in toluene conditions used for the selective deprotection of $O$-benzylsalicylaldehydes ${ }^{24}-$ and furnished the target aldehyde 6 in $85 \%$ yield.

The most common method for the preparation of $O$-triflates consists of treatment of the hydroxylic substrates with $\mathrm{Tf}_{2} \mathrm{O}$ in the presence of organic or inorganic base. ${ }^{12,13,16}$ Hence, upon reaction of 6 with $\mathrm{Tf}_{2} \mathrm{O}$ and TEA in $\mathrm{CH}_{2} \mathrm{Cl}_{2}$ at room temperature and chromatography on silica gel, we obtained 3-triflyloxy-1H-pyrazole-4-carbaldehyde 7 in good yield and purity. No formation of unwanted mixture of $N$ - and $O$-triflated products was detected (Scheme 1).

The triflate 9, which contains a functional ethanone moiety, was obtained by triflation of 4acetyl-1-phenyl-1H-pyrazol-3-ol 8. The latter compound was synthesised using a known synthetic procedure involving the acetylation of 3-hydroxy-1-phenyl-1H-pyrazole following Fries rearrangement conditions $\left(\mathrm{AlCl}_{3}, \mathrm{CS}_{2}\right)\left(\right.$ Scheme 2). ${ }^{18}$

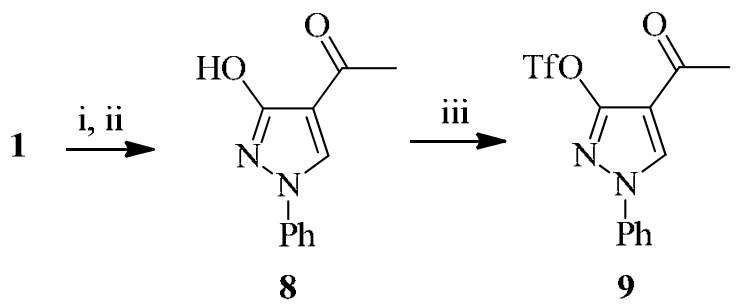

Scheme 2. Reagents and conditions: $\mathrm{i}: \mathrm{Ac}_{2} \mathrm{O}, 100{ }^{\circ} \mathrm{C}, 0.5 \mathrm{~h}, 75 \%$; ii: $\mathrm{AlCl}_{3}, \mathrm{CS}_{2}$, reflux, $3 \mathrm{~h}$, 74\%; iii Tf $2 \mathrm{O}$, TEA, DCM, rt, 1 h, 85\%.

Having successfully prepared pyrazole triflates $\mathbf{7}$ and $\mathbf{9}$, we next examined their ability to participate in palladium-catalysed cross-coupling reactions. The triflates 7 and 9 smoothly underwent the Suzuki-type reaction with phenyl-, 3-chlorophenyl-, 4-(trifluoromethyl)phenyl-, 3-thiophene- and 2-naphthylboronic acids to give compounds 10-14 and 15-19, respectively, in moderate to good yields (50-94\%) (Table 1). In the course of these couplings, $\mathrm{Pd}\left(\mathrm{PPh}_{3}\right)_{4}$ was used as a catalyst and anhydrous $\mathrm{K}_{3} \mathrm{PO}_{4}$ as a base. The reaction was carried out in the presence of $\mathrm{KBr}$, which is known to suppress triflate reduction by stabilizing the cationic ( $\sigma$-aryl)-palladium transition state. ${ }^{25}$ As outlined in Table 1, the final reaction times of triflates 7 and 9 were strongly dependent on the nature of the boronic acid, whereas prolonged reaction times had an opposite correlation with the yields of the target products. The synthesis of carbaldehyde $\mathbf{1 0}$ was reported earlier by a two-step pyrazole ring formation from acetophenone and phenylhydrazine to produce acetophenone phenylhydrazone, followed by Vilsmeier-Haack reaction to give the target carbaldehyde $\mathbf{1 0},{ }^{26}$ which is widely used in the synthesis of biologically active molecules. ${ }^{27}$ Carbaldehyde 14 was similarly obtained from acetonaphthone phenylhydrazone. ${ }^{28}$ 
Standard Sonogashira reaction conditions $\left[\mathrm{Pd}\left(\mathrm{PPh}_{3}\right)_{2} \mathrm{Cl}_{2}, \mathrm{CuI}\right.$, triethylamine] were applied to carry out cross-couplings of the triflates 7 and 9 with phenylacetylene. In both cases, the reaction proceeded smoothly to afford 3-(2-phenylethynyl)-1H-pyrazole-4-carbaldehyde 20 and [3-(2phenylethynyl)-1H-pyrazol-4-yl]ethanone 21, in 70 and $65 \%$ yield, respectively (entries 11,12 , Table 1).

Table 1. Preparation of 3-substituted 1-phenylpyrazole-4-carbaldehydes and the corresponding ethanones

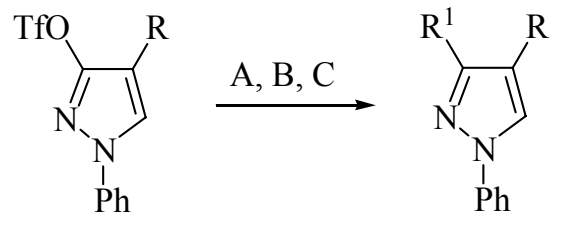

$\begin{array}{ll}7,9 & 10-27\end{array}$

\begin{tabular}{cccccc}
\hline Entry & $\begin{array}{c}\text { Subs } \\
\text { trate }\end{array}$ & Reagent & Conditions $^{\mathrm{a}}$ & $\begin{array}{c}\text { Time } \\
(\mathrm{h})\end{array}$ \\
\hline & $\begin{array}{c}\text { Phenylboronic } \\
\text { acid }\end{array}$ & Yield \\
$(\%)$
\end{tabular}


Table 1. Continued

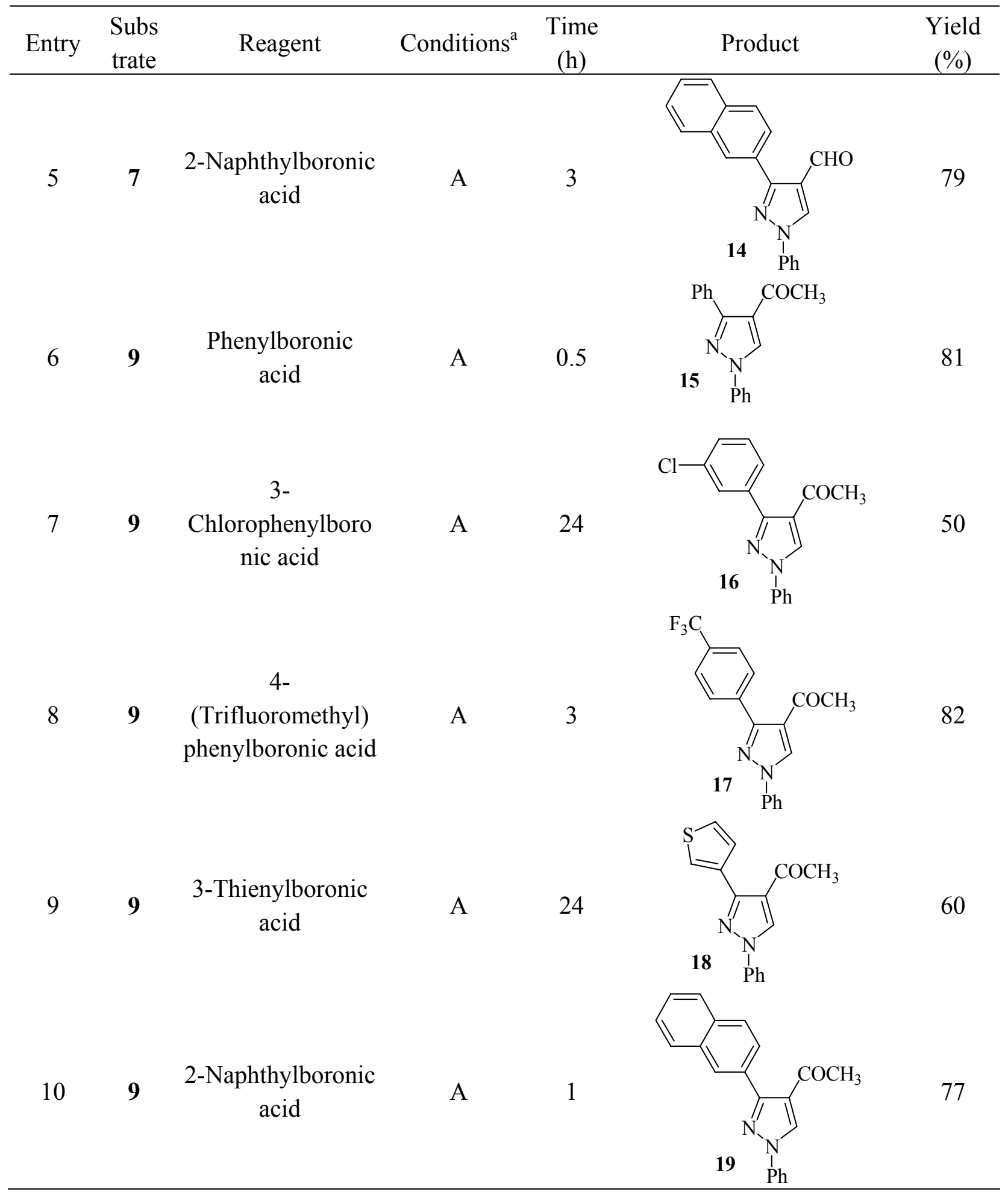


Table 1. Continued

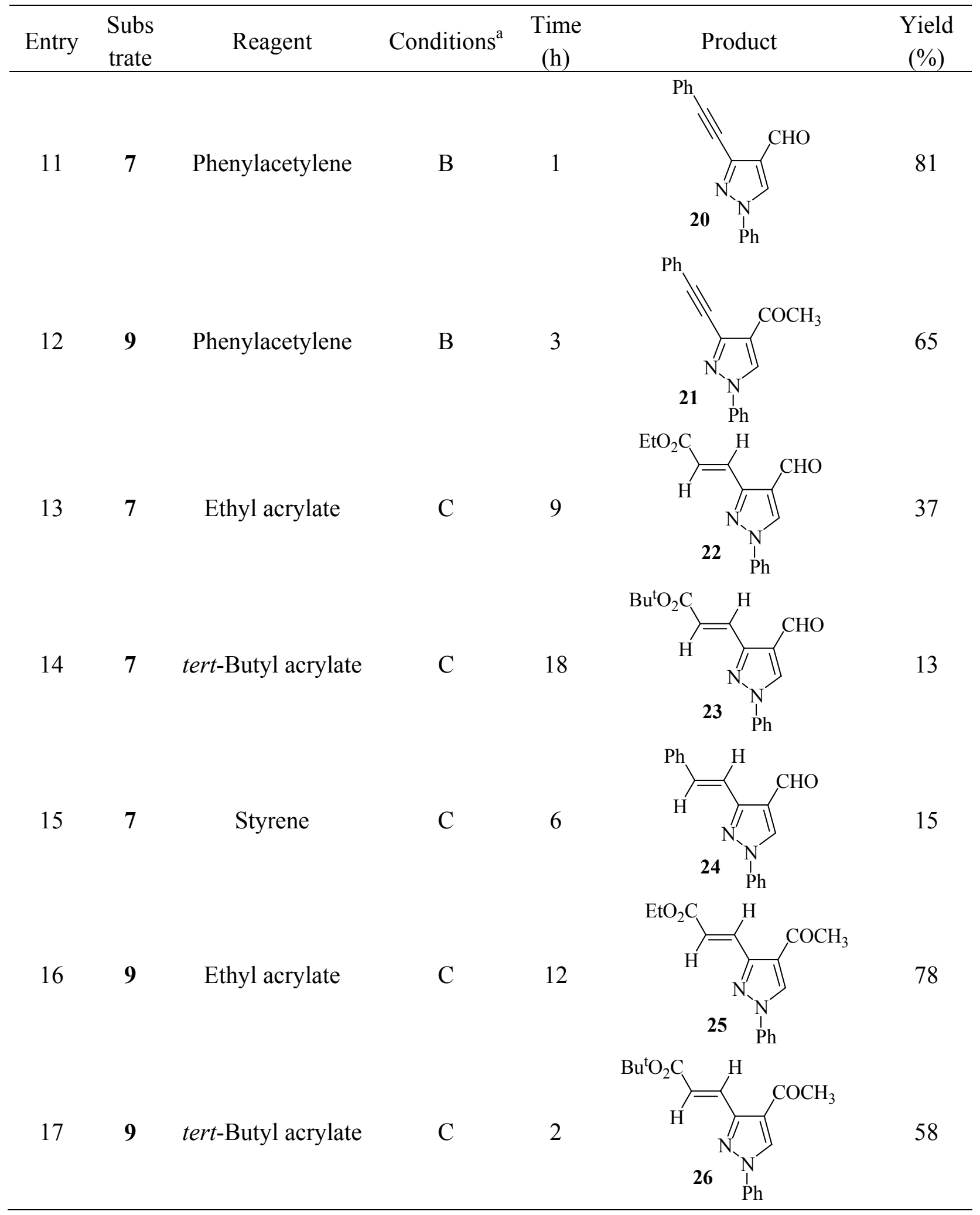


Table 1. Continued

\begin{tabular}{cccccc}
\hline Entry & $\begin{array}{c}\text { Subs } \\
\text { trate }\end{array}$ & Reagent & Conditions $^{\mathrm{a}}$ & $\begin{array}{c}\text { Time } \\
(\mathrm{h})\end{array}$ & Product $^{\text {Yield }}$ \\
$(\%)$
\end{tabular}

${ }^{\mathrm{a}}$ See Section 4.

To evaluate the effectiveness of Heck reactions with triflates 7 and 9, styrene, ethyl and tertbutyl acrylates were representatively employed as coupling partners. The usual Heck reaction conditions $\left[\mathrm{Pd}\left(\mathrm{PPh}_{3}\right)_{2} \mathrm{Cl}_{2}, \quad \mathrm{TEA}\right]$ gave poor yields (13-37\%) of 3-alkenylpyrazole-4carbaldehydes 22-24 (entries 13-15, Table 1), though they were more successful (55-78\%) for the 3-alkenylpyrazolyl ethanones 25-27 (entries 16-18, Table 1). The best yields were obtained by coupling the corresponding triflates with ethyl acrylate. Variations of the reaction parameters such as the catalyst $\left[\mathrm{Pd}\left(\mathrm{PPh}_{3}\right)_{4}, \mathrm{Pd}\left(\mathrm{PPh}_{3}\right)_{2} \mathrm{Cl}_{2}, \mathrm{Pd}(\mathrm{dba})_{3}\right]$ and the base [TEA, NEt $\left.(i \mathrm{Pr})_{2}\right]$, did not result in a marked improvement of yield. The cross-coupled products 22-27 were obtained in an isomerically pure $E$-form after isolation and purification by column chromatography.

The prepared 3,4-difunctionalized pyrazoles are potential synthons in the synthesis of annelated heterocyclic systems. This was briefly demonstrated by treatment of compounds 20 and 21 bearing the phenylethynyl moiety adjacent to the carbonyl group with dry ammonia under elevated temperature and pressure. ${ }^{16}$ The direct formation of pyrazolo[4,3-c]pyridines $\mathbf{2 8}$ and 29 (Scheme 3) was observed in excellent yields.
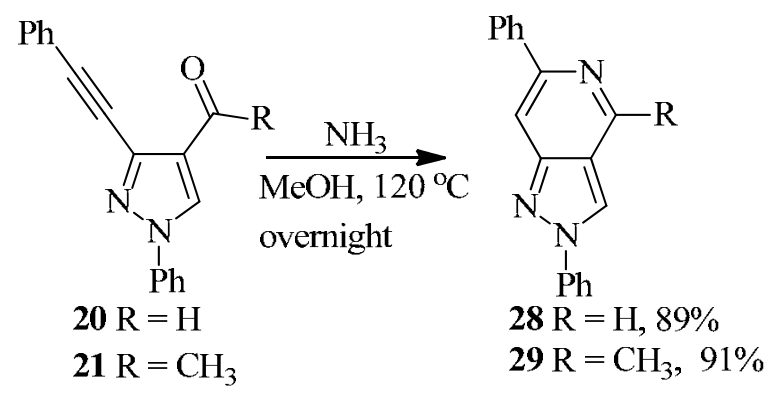

\section{Scheme 3}

Spectral and analytical data for all new compounds were consistent with the given structures. Most newly obtained compounds were subjected to detailed NMR spectroscopic investigations using standard techniques, leading to the unambiguous assignment of chemicals shifts and relevant spin coupling constants (see Experimental). 


\section{Conclusions}

In conclusion, we developed a general approach for the preparation of 3-ethynyl-, 3-ethenyl- and 3-(het)aryl- substituted 1-phenyl-1H-pyrazole-4-carbaldehydes and the corresponding ethanones from 1-phenylpyrazol-3-ol via intermediate triflates by employing Suzuki, Sonogashira and Heck cross-coupling reactions.

\section{Experimental Section}

General. Melting points were determined in capillary tubes on melting point apparatus Electrothermal MEL-TEMP®. Infrared spectra were recorded with Perkin Elmer Spectrum One spectrometer using potassium bromide pellets. ${ }^{1} \mathrm{H}$ NMR spectra were recorded at $300 \mathrm{MHz}$ on a Varian UnityPlus spectrometer and at $500 \mathrm{MHz}$ on a Bruker Avance 500 spectrometer, ${ }^{13} \mathrm{C} \mathrm{NMR}$ spectra were registered at 75 and $125 \mathrm{MHz}$, respectively. Chemical shifts, expressed in ppm, were relative to tetramethylsilane (TMS). ${ }^{15} \mathrm{~N}$ NMR spectra $(50.68 \mathrm{MHz}$, referenced against external nitromethane) and ${ }^{19} \mathrm{~F}$ NMR spectra (470.56 MHz, absolute referencing via $\Xi$ ratio) were obtained on a Bruker Avance 500 instrument with a 'directly' detecting broadband observe probe (BBFO). Mass spectra were recorded using a Waters ZQ instrument (ion spray). Elemental analyses $(\mathrm{C}, \mathrm{H}, \mathrm{N})$ were performed with an Exeter Analytical CE-440 Elemental Analyzer at the Microanalytical Laboratory, Kaunas University of Technology, and were in good agreement $( \pm 0.4 \%)$ with the calculated values. For thin layer chromatographic (TLC) analyses, Merck precoated TLC plates (silica gel 60 F254) were used. Dry THF was distilled from sodium and benzophenone.

\section{Synthetic procedures}

3-(Benzyloxy)-4-bromo-1-phenyl-1H-pyrazole (3). A solution of 4-bromo-3-hydroxy-1phenyl-1H-pyrazole $2^{18}(1.67 \mathrm{~g}, 7 \mathrm{mmol})$ in dry DMF $(20 \mathrm{~mL})$ was cooled to $0{ }^{\circ} \mathrm{C}$ under inert atmosphere and $\mathrm{NaH}$ (60\% dispersion in mineral oil, $280 \mathrm{mg}, 7 \mathrm{mmol})$ was added portion wise. After mixing for $15 \mathrm{~min}$. benzyl chloride $(0.8 \mathrm{~mL}, 7 \mathrm{mmol})$ was added drop wise. The mixture was stirred at $60{ }^{\circ} \mathrm{C}$ for 1 hour, then poured into water and extracted with ethyl acetate. The organic layers were combined, washed with brine, dried over $\mathrm{Na}_{2} \mathrm{SO}_{4}$, filtrated, the solvent was evaporated. The residue was purified by column chromatography $\left(\mathrm{SiO}_{2}\right.$, eluent: ethyl acetate $/ n$ hexane, $1: 7, \mathrm{v} / \mathrm{v})$ to give pure 3. White solid, yield $73 \%, 1.68 \mathrm{~g}, \mathrm{mp} 77-78{ }^{\circ} \mathrm{C}$; IR $\left(v_{\max }, \mathrm{cm}^{-1}\right)$ : $3135\left(\mathrm{CH}_{\text {arom }}\right), 2925\left(\mathrm{CH}_{\text {aliph }}\right), 1598,1543,1495,1360(\mathrm{C}=\mathrm{C}, \mathrm{C}-\mathrm{N}), 1218,1097(\mathrm{C}-\mathrm{O}), 754$, 745, 687, $674\left(\mathrm{CH}=\mathrm{CH}\right.$ of monosubstituted benzenes). ${ }^{1} \mathrm{H} \mathrm{NMR}\left(300 \mathrm{MHz}, \mathrm{CDCl}_{3}\right): \delta_{\mathrm{H}} 5.41(\mathrm{~s}$, 2H, $\mathrm{CH}_{2}$ ), 7.25 (m, 1H, NPh H-4), 7.37 (m, 1H, CPh H-4), 7.42 (m, 2H, CPh H-3,5), 7.44 (m, 2H, NPh H-3,5), 7.55 (m, 2H, CPh H-2,6), 7.58 (m, 2H, NPh H-2,6), 7.79 (s, 1H, H-5). ${ }^{13} \mathrm{C}$ NMR $\left(75 \mathrm{MHz}, \mathrm{CDCl}_{3}\right): \delta_{\mathrm{C}} 71.1\left(\mathrm{CH}_{2},{ }^{1} J=147.0 \mathrm{~Hz},{ }^{3} J\left(\mathrm{OCH}_{2}, \mathrm{CPhH} 2,6\right)=4.6 \mathrm{~Hz}\right), 82.5(\mathrm{C}-4$, $\left.{ }^{2} J(\mathrm{C} 4, \mathrm{H} 5)=5.2 \mathrm{~Hz}\right), 117.6(\mathrm{NPh} \mathrm{C}-2,6), 125.7$ (NPh C-4), $127.7\left(\mathrm{C}-5,{ }^{1} J=192.0 \mathrm{~Hz}\right), 128.0$ 
(CPh C-2,4,6), 128.4 (CPh C-3,5), 129.4 (NPh C-3,5), 136.7 (CPh C-1), 139.7 (NPh C-1), 160.6 $\left(\mathrm{C}-3,{ }^{3} J(\mathrm{C} 3, \mathrm{H} 5)=8.8 \mathrm{~Hz},{ }^{3} J\left(\mathrm{C} 3, \mathrm{OCH}_{2}\right)=2.7 \mathrm{~Hz}\right)$; MS $m / z(\%): 353 / 351\left([\mathrm{M}+\mathrm{Na}]^{+}, 100\right)$. Anal. Calcd for $\mathrm{C}_{16} \mathrm{H}_{13} \mathrm{BrN}_{2} \mathrm{O}: \mathrm{C}, 58.38 ; \mathrm{H}, 3.98 ; \mathrm{N}, 8.51$. Found: C, 58.70; H, 4.07; N, 8.23.

3-(Benzyloxy)-1-phenyl-1H-pyrazole-4-carbaldehyde (4). Method iii. To a solution of 3 (656 $\mathrm{mg}, 2 \mathrm{mmol})$ in $16 \mathrm{~mL}$ of dry THF under inert atmosphere at $-78^{\circ} \mathrm{C} n$-BuLi $(2.5 \mathrm{M}$ in hexane, $0.8 \mathrm{~mL}, 2 \mathrm{mmol})$ was added drop wise and dry DMF $(0.23 \mathrm{~mL}, 3 \mathrm{mmol})$ was added. The mixture was gradually warmed up to room temperature and stirred for $30 \mathrm{~min}$. The reaction mixture was poured into water and extracted with ethyl acetate. The organic layers were combined, washed with brine, dried over $\mathrm{Na}_{2} \mathrm{SO}_{4}$, filtrated, the solvent was evaporated. The residue was purified by column chromatography $\left(\mathrm{SiO}_{2}\right.$, eluent: ethyl acetate/n-hexane, 1:5, v/v) to give pure 4 . White solid, yield 70\%, $389 \mathrm{mg}$.

Method iv. Phosphorus oxychloride $(0.73 \mathrm{~mL}, 8 \mathrm{mmol})$ was added dropwise to dry DMF $(0.61$ $\mathrm{mL}, 8 \mathrm{mmol})$ at $-10^{\circ} \mathrm{C}$. Then $5(500 \mathrm{mg}, 2 \mathrm{mmol})$ was added to the Vilsmeier-Haack complex and the reaction mixture heated at $70{ }^{\circ} \mathrm{C}$ for $12 \mathrm{~h}$. After neutralization with $10 \%$ aq $\mathrm{NaHCO}_{3}$, the preticipate was filtered of and recrystallized from dichloromethane. Yield 60\%, $334 \mathrm{mg}, \mathrm{mp} 151$ $153{ }^{\circ} \mathrm{C}$ (lit. mp 153-154 $\left.{ }^{\circ} \mathrm{C}\right) ;^{19 \mathrm{a}} \mathrm{IR}\left(v_{\max }, \mathrm{cm}^{-1}\right): 3094\left(\mathrm{CH}_{\text {arom }}\right), 2957\left(\mathrm{CH}_{\text {aliph }}\right), 1667(\mathrm{C}=\mathrm{O}), 1559$, 1504, $1360(\mathrm{C}=\mathrm{C}, \mathrm{C}-\mathrm{N}), 1222,1203(\mathrm{C}-\mathrm{O}), 753,735,687,677(\mathrm{CH}=\mathrm{CH}$ of monosubstituted benzenes), ${ }^{1} \mathrm{H}$ NMR $\left(300 \mathrm{MHz}, \mathrm{CDCl}_{3}\right): \delta_{\mathrm{H}} 5.46\left(\mathrm{~s}, 2 \mathrm{H}, \mathrm{CH}_{2}\right), 7.34(\mathrm{~m}, 1 \mathrm{H}, \mathrm{NPh} \mathrm{H}-4), 7.38(\mathrm{~m}$, 1H, CPh H-4), 7.42 (m, 2H, CPh H-3,5), 7.48 (m, 2H, NPh H-3,5), 7.53 (m, 2H, CPh H-2,6), $7.66(\mathrm{~m}, 2 \mathrm{H}, \mathrm{NPh} \mathrm{H}-2,6), 8.27$ (s, 1H, H-5), 9.89 (CHO). ${ }^{13} \mathrm{C} \mathrm{NMR}\left(75 \mathrm{MHz}, \mathrm{CDCl}_{3}\right): \delta_{\mathrm{C}} 71.1$ $\left(\mathrm{CH}_{2},{ }^{1} J=147.4 \mathrm{~Hz},{ }^{3} J\left(\mathrm{OCH}_{2}, \mathrm{CPhH}-2,6\right)=4.3 \mathrm{~Hz}\right), 111.5\left(\mathrm{C}-4,{ }^{2} J(\mathrm{C} 4, \mathrm{CHO})=25.0 \mathrm{~Hz}\right.$, $\left.{ }^{2} J(\mathrm{C} 4, \mathrm{H} 5)=6.9 \mathrm{~Hz}\right), 118.8(\mathrm{NPh} \mathrm{C}-2,6), 127.2(\mathrm{NPh} \mathrm{C}-4), 128.2$ (CPh C-2,6), 128.3 (CPh C-4), 128.5 (CPh C-3,5), $129.3\left(\mathrm{C}-5,{ }^{1} J=190.1 \mathrm{~Hz},{ }^{3} J(\mathrm{C} 5, \mathrm{CHO})=5.1 \mathrm{~Hz}\right), 129.6(\mathrm{NPh} \mathrm{C}-3,5), 136.2$ (CPh C-1), 139.0 (NPh C-1), $163.6\left(\mathrm{C}-3,{ }^{3} J(\mathrm{C} 3, \mathrm{H} 5)=8.8 \mathrm{~Hz},{ }^{3} J\left(\mathrm{C} 3, \mathrm{OCH}_{2}\right)=2.7 \mathrm{~Hz}\right), 183.3$ $\left(\mathrm{CHO},{ }^{1} J=176.5 \mathrm{~Hz}\right)$; MS $m / z(\%): 301\left([\mathrm{M}+\mathrm{Na}]^{+}, 100\right)$; Anal. Calcd for $\mathrm{C}_{17} \mathrm{H}_{14} \mathrm{~N}_{2} \mathrm{O}_{2} \bullet 0.5 \mathrm{H}_{2} \mathrm{O}$ : C, 71.07; H, 5.26; N, 9.75. Found: C, 71.09; H, 5.14; N, 9.59.

3-(Benzyloxy)-1-phenyl-1H-pyrazole (5). This compound was synthesized in analogy to compound 3 from 3-hydroxy-1-phenyl-1H-pyrazole 1 (1.12 g, $7 \mathrm{mmol}$ ). White solid, yield 85\%, $1.49 \mathrm{~g}, \mathrm{mp} 71{ }^{\circ} \mathrm{C}$; IR $\left(v_{\max }, \mathrm{cm}^{-1}\right): 3035\left(\mathrm{CH}_{\text {arom }}\right), 2954\left(\mathrm{CH}_{\text {aliph }}\right), 1547,1478,1359(\mathrm{C}=\mathrm{C}, \mathrm{C}-\mathrm{N})$, 1048, $1019(\mathrm{C}-\mathrm{O}), 758,737,699,689\left(\mathrm{CH}=\mathrm{CH}\right.$ of monosubstituted benzenes). ${ }^{1} \mathrm{H}$ NMR (300 $\mathrm{MHz}_{\mathrm{CDCl}}$ ): $\delta_{\mathrm{H}} 5.34\left(\mathrm{~s}, 2 \mathrm{H}, \mathrm{CH}_{2}\right), 5.94(\mathrm{~d}, J=2.7 \mathrm{~Hz}, 1 \mathrm{H}, \mathrm{H}-4), 7.22(\mathrm{~m}, 1 \mathrm{H}, \mathrm{CPh} \mathrm{H}-4), 7.36$ (m, 1H, NPh H-4), 7.40 (m, 2H, CPh H-3,5), 7.43 (m, 2H, NPh H-3,5), 7.51 (m, 2H, CPh H-2,6), $7.63(\mathrm{~m}, 2 \mathrm{H}, \mathrm{NPh} \mathrm{H}-2,6), 7.75(\mathrm{~d}, J=2.7 \mathrm{~Hz}, 1 \mathrm{H}, \mathrm{H}-5) .{ }^{13} \mathrm{C} \mathrm{NMR}\left(75 \mathrm{MHz}, \mathrm{CDCl}_{3}\right): \delta_{\mathrm{C}} 70.9$ $\left(\mathrm{CH}_{2},{ }^{1} \mathrm{~J}=146.3 \mathrm{~Hz},{ }^{3} \mathrm{~J}\left(\mathrm{OCH}_{2}, \mathrm{CPhH} 2,6\right)=4.6 \mathrm{~Hz}\right), 94.1\left(\mathrm{C}-4,{ }^{1} \mathrm{~J}=179.8 \mathrm{~Hz},{ }^{2} J(\mathrm{C} 4, \mathrm{H} 5)=8.2 \mathrm{~Hz}\right)$, 117.8 (NPh C-2,6), 125.3 (CPh C-4), 127.7 (C-5, $\left.{ }^{1} J=186.3 \mathrm{~Hz},{ }^{2} J(\mathrm{C} 5, \mathrm{H} 4)=8.6 \mathrm{~Hz}\right), 128.0(\mathrm{NPh}$ $\mathrm{C}-4), 128.0\left(\mathrm{CPh} \mathrm{C}-2,6,{ }^{3} J\left(\mathrm{C}, \mathrm{OCH}_{2}\right)=4.4 \mathrm{~Hz}\right), 128.4(\mathrm{CPh} \mathrm{C}-3,5), 129.3(\mathrm{NPh} \mathrm{C}-3,5), 137.0$ $\left(\mathrm{CPh} \mathrm{C}-1,{ }^{2} J\left(\mathrm{C}, \mathrm{OCH}_{2}\right)=3.9 \mathrm{~Hz}\right), 140.2(\mathrm{NPh} \mathrm{C}-1), 164.3 \quad\left(\mathrm{C}-3,{ }^{2} J(\mathrm{C} 3, \mathrm{H} 4)=179.8 \mathrm{~Hz}\right.$, $\left.{ }^{3} J(\mathrm{C} 3, \mathrm{H} 5)=10.4 \mathrm{~Hz},{ }^{3} J\left(\mathrm{C}_{3}, \mathrm{OCH}_{2}\right)=2.5 \mathrm{~Hz}\right)$; MS $m / z(\%): 274\left([\mathrm{M}+\mathrm{Na}]^{+}, 100\right)$. Anal. Calcd for $\mathrm{C}_{16} \mathrm{H}_{14} \mathrm{~N}_{2} \mathrm{O}$ : C, 76.78; H, 5.64; N, 11.19. Found: C, 76.89; H, 5.66; N, 11.23. 
3-Hydroxy-1-phenyl-1H-pyrazole-4-carbaldehyde (6). To a solution of 4 (556 $\mathrm{mg}, 2 \mathrm{mmol})$ in toluene $(10 \mathrm{~mL})$ trifluoroacetic acid $(10 \mathrm{~mL})$ was added. The mixture was stirred for 2 days at $\mathrm{rt}$ Toluene and trifluoroacetic acid were evaporated. The residue was purified by column chromatography $\left(\mathrm{SiO}_{2}\right.$, eluent: ethyl acetate/n-hexane, $\left.1: 1, \mathrm{v} / \mathrm{v}\right)$ to give pure 6. Pale solid, yield

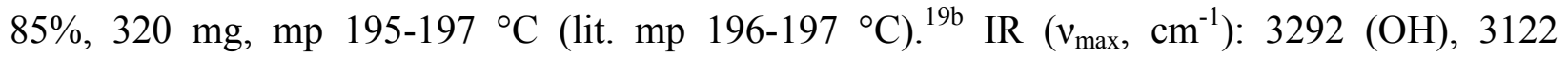
$\left(\mathrm{CH}_{\text {arom}}\right), 1671(\mathrm{C}=\mathrm{O}), 1597,1578,1509,1500,1442(\mathrm{C}=\mathrm{C}, \mathrm{C}-\mathrm{N}), 1284,1214,1186,1170(\mathrm{C}-$ $\mathrm{O}), 760,685\left(\mathrm{CH}=\mathrm{CH}\right.$ of monosubstituted benzene). ${ }^{1} \mathrm{H}$ NMR $\left(300 \mathrm{MHz}, \mathrm{DMSO}-d_{6}\right): \delta_{\mathrm{H}} 7.31$ (m, 1H, Ph H-4), 7.48 (m, 2H, Ph H-3,5), 7.80 (m, 2H, Ph H-2,6), 8.91 (s, 1H, H-5), 9.75 (s, 1H, $\mathrm{CHO}), 11.51$ (br s, $1 \mathrm{H}, \mathrm{OH}) .{ }^{13} \mathrm{C}$ NMR $\left(75 \mathrm{MHz}, \mathrm{DMSO}-d_{6}\right): \delta_{\mathrm{C}} 111.0\left(\mathrm{C}-4,{ }^{2} \mathrm{~J}(\mathrm{C} 4, \mathrm{CHO})=25.0\right.$ $\left.\mathrm{Hz},{ }^{2} J(\mathrm{C} 4, \mathrm{H} 5)=7.0 \mathrm{~Hz}\right), 118.2(\mathrm{Ph} \mathrm{C}-2,6), 126.7$ (Ph C-4), 129.5 (Ph C-3,5), $131.0\left(\mathrm{C}-5,{ }^{1} J=\right.$ $174.4 \mathrm{~Hz}), 138.7(\mathrm{Ph} \mathrm{C}-1), 162.4\left(\mathrm{C}-3,{ }^{3} \mathrm{~J}(\mathrm{C} 3, \mathrm{H} 5)=8.9 \mathrm{~Hz},{ }^{3} J(\mathrm{C} 3, \mathrm{CHO})=1.8 \mathrm{~Hz}\right), 183.2(\mathrm{CHO})$; MS $m / z(\%): 211\left([\mathrm{M}+\mathrm{Na}]^{+}, 100\right)$; Anal. Calcd for $\mathrm{C}_{10} \mathrm{H}_{8} \mathrm{~N}_{2} \mathrm{O}_{2}: \mathrm{C}, 63.82 ; \mathrm{H}, 4.28 ; \mathrm{N}, 14.89$. Found: C, 64.01; H, 4.26; N, 14.52.

4-Formyl-1-phenyl-1H-pyrazol-3-yl trifluoromethanesulfonate (7). Pyrazole 6 (1.13 g, 6.0 mmol), trifluormethansulfonic anhydride $(1 \mathrm{~mL}, 6 \mathrm{mmol})$ and triethylamine $(1 \mathrm{~mL}, 7.2 \mathrm{mmol})$ were dissolved in dichloromethane $(20 \mathrm{~mL})$ and the mixture was stirred at $\mathrm{rt}$ for 1 hour. The reaction mixture was poured into water and extracted with ethyl acetate. Combined organic layers were washed with brine and dried over $\mathrm{Na}_{2} \mathrm{SO}_{4}$, the solvent was evaporated. The residue was purified by flash chromatography $\left(\mathrm{SiO}_{2}\right.$, eluent: ethyl acetate/ $n$-hexane, 1:4, v/v). Pale solid, yield $83 \%, 1.59 \mathrm{~g}, \mathrm{mp} 73-74{ }^{\circ} \mathrm{C}$; IR $\left(v_{\max }, \mathrm{cm}^{-1}\right): 3130\left(\mathrm{CH}_{\text {arom }}\right), 1681(\mathrm{C}=\mathrm{O}), 1557,1458,1429$, $1393(\mathrm{C}=\mathrm{C}, \mathrm{C}-\mathrm{N}), 1247,1228,1204,1136(\mathrm{C}-\mathrm{O}, \mathrm{C}-\mathrm{F}, \mathrm{S}=\mathrm{O}), 763,687(\mathrm{CH}=\mathrm{CH}$ of monosubstituted benzene). ${ }^{1} \mathrm{H}$ NMR $\left(300 \mathrm{MHz}, \mathrm{CDCl}_{3}\right): \delta_{\mathrm{H}} 7.44(\mathrm{~m}, 1 \mathrm{H}, \mathrm{Ph} \mathrm{H}-4), 7.53(\mathrm{~m}, 2 \mathrm{H}$, $\mathrm{Ph} \mathrm{H}-3,5), 7.67$ (m, 2H, Ph H-2,6), 8.41 (s, 1H, H-5), 9.92 (s, 1H, CHO). ${ }^{13} \mathrm{C}$ NMR (75 MHz, $\left.\mathrm{CDCl}_{3}\right): \delta_{\mathrm{C}} 114.7\left(\mathrm{C}-4,{ }^{2} J(\mathrm{C} 4, \mathrm{H} 5)=7.0 \mathrm{~Hz},{ }^{2} J(\mathrm{C} 4, \mathrm{CHO})=27.1 \mathrm{~Hz}\right), 118.6\left(\mathrm{CF}_{3},{ }^{1} \mathrm{~J}=321.2 \mathrm{~Hz}\right)$, 119.5 (Ph C-2,6), 128.8 (Ph C-4), 129.9 (Ph C-3,5), 130.9 (C-5, ${ }^{1} J=193.3 \mathrm{~Hz},{ }^{2} J(\mathrm{C} 5, \mathrm{CHO})=3.3$ $\mathrm{Hz}), 138.2$ (Ph C-1), $152.3\left(\mathrm{C}-3,{ }^{3} J(\mathrm{C} 3, \mathrm{H} 5)=10.3 \mathrm{~Hz},{ }^{3} \mathrm{~J}(\mathrm{C} 3, \mathrm{CHO})=2.2 \mathrm{~Hz}\right), 181.0(\mathrm{CHO}),{ }^{19} \mathrm{~F}$ NMR (470 MHz, $\left.\mathrm{CDCl}_{3}\right): \delta_{\mathrm{F}}-72.0\left(\mathrm{CF}_{3}\right) ; \mathrm{MS} m / z(\%): 321\left([\mathrm{M}+\mathrm{H}]^{+}, 100\right)$. Anal. Calcd for $\mathrm{C}_{11} \mathrm{H}_{7} \mathrm{~F}_{3} \mathrm{~N}_{2} \mathrm{O}_{4} \mathrm{~S}$ : C, 41.26; H, 2.20; N, 8.75. Found: C, 41.04; H, 2.16; N, 8.56.

4-Acetyl-1-phenyl-1H-pyrazol-3-yl trifluoromethanesulfonate (9). This compound was synthesized in analogy to compound 7 synthesis using pyrazole 8 (1.21 g, $6 \mathrm{mmol})$. White crystals, yield $85 \%, 1.70 \mathrm{~g}, \mathrm{mp} 89^{\circ} \mathrm{C}$; IR $\left(v_{\max }, \mathrm{cm}^{-1}\right): 3137\left(\mathrm{CH}_{\text {arom }}\right), 1678(\mathrm{C}=\mathrm{O}), 1556,1427$ $(\mathrm{C}=\mathrm{C}, \mathrm{C}-\mathrm{N}), 1275,1240,1211,1135(\mathrm{C}-\mathrm{O}, \mathrm{C}-\mathrm{F}, \mathrm{S}=\mathrm{O}), 745,712(\mathrm{CH}=\mathrm{CH}$ of monosubstituted benzene). ${ }^{1} \mathrm{H}$ NMR $\left(300 \mathrm{MHz}, \mathrm{CDCl}_{3}\right): \delta_{\mathrm{H}} 2.53\left(\mathrm{~s}, 3 \mathrm{H}, \mathrm{CH}_{3}\right), 7.41(\mathrm{~m}, 1 \mathrm{H}, \mathrm{Ph} \mathrm{H}-4), 7.51(\mathrm{~m}, 2 \mathrm{H}$, $\mathrm{Ph} \mathrm{H}-3,5), 7.66$ (m, 2H, Ph H-2,6), 8.35 (s, 1H, H-5). ${ }^{13} \mathrm{C} \mathrm{NMR}\left(75 \mathrm{MHz}, \mathrm{CDCl}_{3}\right): \delta_{\mathrm{C}} 28.7\left(\mathrm{CH}_{3}\right.$, $\left.{ }^{1} J=128.1 \mathrm{~Hz}\right), 115.0\left(\mathrm{C}-4,{ }^{2} J(\mathrm{C} 4, \mathrm{H} 5)=6.9 \mathrm{~Hz},{ }^{3} J\left(\mathrm{C}_{4}, \mathrm{CH}_{3}\right)=1.5 \mathrm{~Hz}\right), 118.6\left(\mathrm{CF}_{3},{ }^{1} J=321.2 \mathrm{~Hz}\right)$, 119.4 (Ph C-2,6), 128.5 (Ph C-4), 129.8 (Ph C-3,5), 131.0 (C-5, ${ }^{1} J=192.6$ Hz), 138.3 (Ph C-1), $151.3\left(\mathrm{C}-3,{ }^{3} J(\mathrm{C} 3, \mathrm{H} 5)=10.7 \mathrm{~Hz}\right), 189.3\left(\mathrm{CO},{ }^{2} J\left(\mathrm{CO}, \mathrm{CH}_{3}\right)=6.2 \mathrm{~Hz}\right) .{ }^{15} \mathrm{~N} \mathrm{NMR}\left(50 \mathrm{MHz}, \mathrm{CDCl}_{3}\right)$ : $\delta_{\mathrm{N}}-173.8(\mathrm{~N}-1), \mathrm{N}-2$ was not found. ${ }^{19} \mathrm{~F} \mathrm{NMR}\left(470 \mathrm{MHz}, \mathrm{CDCl}_{3}\right): \delta_{\mathrm{F}}-72.3\left(\mathrm{CF}_{3}\right) ; \mathrm{MS} \mathrm{m} / z(\%)$ : $335\left([\mathrm{M}+\mathrm{H}]^{+}\right.$, 100). Anal. Calcd for $\mathrm{C}_{12} \mathrm{H}_{9} \mathrm{~F}_{3} \mathrm{~N}_{2} \mathrm{O}_{4} \mathrm{~S}: \mathrm{C}, 43.12 ; \mathrm{H}, 2.71 ; \mathrm{N}, 8.38$. Found: C, $43.51 ; \mathrm{H}, 2.78 ; \mathrm{N}, 8.53$. 
General procedure for the preparation of 4-substituted 1-phenyl-1H-pyrazol-4carbaldehydes and ketones by Suzuki-Miyaura cross-coupling reaction: method A

To a solution of appropriate pyrazole 7 or $9(0.5 \mathrm{mmol})$ in 1,4-dioxane $(5 \mathrm{~mL})$ under argon atmosphere anhydrous $\mathrm{K}_{3} \mathrm{PO}_{4}(318 \mathrm{mg}, 1.5 \mathrm{mmol})$, an appropriate boronic acid $(1.5 \mathrm{mmol})$, $\mathrm{Pd}\left(\mathrm{PPh}_{3}\right)_{4}(46 \mathrm{mg}, 0.04 \mathrm{mmol})$ and $\mathrm{KBr}(66 \mathrm{mg}, 0.55 \mathrm{mmol})$ were added. After refluxing for the given time under argon atmosphere the mixture was diluted with water and the extraction was done with ethyl acetate. The combined organic layers were washed with brine, dried over $\mathrm{Na}_{2} \mathrm{SO}_{4}$, filtrated, the solvent was evaporated. The residue was purified by flash chromatography $\left(\mathrm{SiO}_{2}\right.$, eluent: ethyl acetate $/ n$-hexane, $\left.1: 4, \mathrm{v} / \mathrm{v}\right)$ to yield compounds 10-19.

1,3-Diphenyl-1H-pyrazole-4-carbaldehyde (10). White solid, Yield 94\%, $117 \mathrm{mg}, \mathrm{mp}$ 142-144 ${ }^{\circ} \mathrm{C}$ (lit. mp 137-145 $\left.{ }^{\circ} \mathrm{C}\right) .{ }^{27} \mathrm{IR}\left(v_{\max }, \mathrm{cm}^{-1}\right): 3126\left(\mathrm{CH}_{\text {arom }}\right), 2839\left(\mathrm{CH}_{\text {aliph }}\right), 1674(\mathrm{C}=\mathrm{O}), 1525$, $1510(\mathrm{C}=\mathrm{C}, \mathrm{C}-\mathrm{N}), 772,753,699,688(\mathrm{CH}=\mathrm{CH}$ of monosubstituted benzenes $) .{ }^{1} \mathrm{H}$ NMR $(300$ $\left.\mathrm{MHz} \mathrm{CDCl}_{3}\right): \delta_{\mathrm{H}} 7.40(\mathrm{~m}, 1 \mathrm{H}, \mathrm{NPh} \mathrm{H}-4), 7.47-7.55(\mathrm{~m}, 5 \mathrm{H}, \mathrm{CPh} \mathrm{H}-3,4,5$ and NPh H-3,5), 7.80 (m, 2H, NPh H-2,6), 7.83 (m, 2H, CPh H-2,6), 8.55 (s, 1H, H-5), 10.06 (s, 1H, CHO). ${ }^{13} \mathrm{C}$ NMR $\left(75 \mathrm{MHz}, \mathrm{CDCl}_{3}\right): \delta_{\mathrm{C}} 119.8(\mathrm{NPh} \mathrm{C}-2,6), 122.6\left(\mathrm{C}-4,{ }^{2} J(\mathrm{C} 4, \mathrm{C} 5)=7.0 \mathrm{~Hz},{ }^{2} J(\mathrm{C} 4, \mathrm{CHO})=25.7 \mathrm{~Hz}\right)$, 128.0 (NPh C-4), 128.7 (CPh C-3,5), 129.0 (CPh C-2,6), 129.3 (CPh C-4), 129.7 (NPh C-3,5), $130.9\left(\mathrm{C}-5,{ }^{1} \mathrm{~J}=190.1 \mathrm{~Hz},{ }^{3} \mathrm{~J}(\mathrm{C} 5, \mathrm{CHO})=4.6 \mathrm{~Hz}\right), 131.4(\mathrm{CPh} \mathrm{C}-1), 139.0(\mathrm{NPh} \mathrm{C}-1), 154.8(\mathrm{C}-3$, $\left.{ }^{3} J(\mathrm{C} 3, \mathrm{H} 5)=7.2 \mathrm{~Hz}\right), 185.1\left(\mathrm{CHO},{ }^{1} J=176.1 \mathrm{~Hz},{ }^{3} J(\mathrm{CHO}, \mathrm{H} 5)=1.0 \mathrm{~Hz}\right) ; \mathrm{MS} \mathrm{m} / z(\%): 249$ $\left([\mathrm{M}+\mathrm{H}]^{+}\right.$, 100). Anal. Calcd for $\mathrm{C}_{16} \mathrm{H}_{12} \mathrm{~N}_{2} \mathrm{O} \bullet 0.2 \mathrm{H}_{2} \mathrm{O}: \mathrm{C}, 76.29 ; \mathrm{H}, 4.96 ; \mathrm{N}, 11.12$. Found: $\mathrm{C}$, 76.67; H, 5.01; N, 10.85 .

3-(3-Chlorophenyl)-1-phenyl-1H-pyrazole-4-carbaldehyde (11). White solid, yield 82\%, 116 mg, mp 99-101 ${ }^{\circ} \mathrm{C}$; IR $\left(v_{\max }, \mathrm{cm}^{-1}\right): 3113\left(\mathrm{CH}_{\text {arom }}\right), 1683(\mathrm{C}=\mathrm{O}), 759,747,687(\mathrm{CH}=\mathrm{CH}$ of mono- and disubstituted benzene). ${ }^{1} \mathrm{H}$ NMR $\left(300 \mathrm{MHz}, \mathrm{CDCl}_{3}\right): \delta_{\mathrm{H}} 7.40(\mathrm{~m}, 1 \mathrm{H}, \mathrm{NPh} \mathrm{H}-4), 7.42$ (m, 1H, 3ClPh H-4), 7.43 (m, 1H, 3ClPh H-5), 7.51 (m, 2H, NPh H-3,5), 7.75 (m, 1H, 3ClPh H6), 7.78 (m, 2H, NPh H-2,6), 7.88 (s, 1H, 3ClPh H-2), 8.53 (s, 1H, H-5), 10.03 (s, 1H, CHO); ${ }^{13} \mathrm{C}$ NMR $\left(75 \mathrm{MHz}, \mathrm{CDCl}_{3}\right): \delta_{\mathrm{C}} 119.7(\mathrm{NPh} \mathrm{C}-2,6), 122.5 \quad\left(\mathrm{C}-4,{ }^{2} J(\mathrm{C} 4, \mathrm{CHO})=25.8 \mathrm{~Hz}\right.$, $\left.{ }^{3} J(\mathrm{C} 4, \mathrm{H} 5)=7.0 \mathrm{~Hz}\right), 127.1$ (3ClPh C-6), 128.1 (NPh C-4), 128.8 (3ClPh C-2), 129.3 (3ClPh C-4), 129.7 (NPh C-3,5), 129.9 (3ClPh C-5), 131.8 (C-5, $\left.{ }^{1} J=190.3,{ }^{3} J(\mathrm{C} 5, \mathrm{CHO})=4.1\right), 133.0(3 \mathrm{ClPh}$ $\mathrm{C}-1$ ), 134.6 (3ClPh C-3), 138.8 (NPh C-1), 152.9 (C-3, $\left.{ }^{3} J(\mathrm{C} 3, \mathrm{H} 5)=7.3 \mathrm{~Hz},{ }^{3} J(\mathrm{C} 3, \mathrm{CHO})=1.7 \mathrm{~Hz}\right)$, $184.5\left(\mathrm{CHO},{ }^{1} J=175.9 \mathrm{~Hz},{ }^{3} J(\mathrm{CHO}, \mathrm{H} 5)=1.0 \mathrm{~Hz}\right) ;{ }^{15} \mathrm{~N}$ NMR $\left(50 \mathrm{MHz}, \mathrm{CDCl}_{3}\right): \delta_{\mathrm{N}}-159.9(\mathrm{~N}-$ 1), N-2 was not found; MS $m / z$ (\%): 285/283 ([M+H ] , 37/100). Anal. Calcd for $\mathrm{C}_{16} \mathrm{H}_{11} \mathrm{ClN}_{2} \mathrm{O}$ : C, 67.97; H, 3.92; N, 9.91. Found: C, 68.09; H, 4.12; N, 9.56.

3-[4-(Trifluoromethyl)phenyl]-1-phenyl-1H-pyrazole-4-carbaldehyde (12). White solid, yield 60\%, $95 \mathrm{mg}, \mathrm{mp} 144{ }^{\circ} \mathrm{C}$; IR $\left(v_{\max }, \mathrm{cm}^{-1}\right): 3130\left(\mathrm{CH}_{\text {arom }}\right), 1678(\mathrm{C}=\mathrm{O}), 1523(\mathrm{C}=\mathrm{C}, \mathrm{C}-\mathrm{N}), 856$, 760, $686\left(\mathrm{CH}=\mathrm{CH}\right.$ of mono- and disubstituted benzenes). ${ }^{1} \mathrm{H}$ NMR $\left(500 \mathrm{MHz}, \mathrm{CDCl}_{3}\right): \delta_{\mathrm{H}} 7.42$ (m, 2H, NPh H-4), 7.54 (m, 2H, NPh H-3,5), 7.76 (m, 2H, 4CF 3 Ph H-3,5), 7.80 (m, 2H, NPh H2,6), 8.03 (m, 2H, 4CF 3 Ph H-2,6), 8.57 (s, 1H, H-5), 10.07 (s, 1H, CHO). ${ }^{13} \mathrm{C} \mathrm{NMR} \mathrm{(125} \mathrm{MHz,}$ $\left.\mathrm{CDCl}_{3}\right): \delta_{\mathrm{C}} 119.8(\mathrm{NPh} \mathrm{C}-2,6), 122.7\left(\mathrm{C}-4,{ }^{2} J(\mathrm{C} 4, \mathrm{CHO})=26.0 \mathrm{~Hz},{ }^{2} J(\mathrm{C} 4, \mathrm{H} 5)=7.0 \mathrm{~Hz}\right), 124.0$ $\left(\mathrm{CF}_{3},{ }^{1} J=272.3 \mathrm{~Hz}\right), 125.6\left(4 \mathrm{CF}_{3} \mathrm{Ph} \mathrm{C}-3,5\right), 128.2(\mathrm{NPh} \mathrm{C}-4), 129.2\left(4 \mathrm{CF}_{3} \mathrm{Ph} \mathrm{C}-2,6\right), 129.8$ (NPh C-3,5), 131.1 (4CF 3 Ph C-4), $132.4\left(\mathrm{C}-5,{ }^{1} \mathrm{~J}=190.1,{ }^{3} \mathrm{~J}(\mathrm{C} 5, \mathrm{CHO})=3.8 \mathrm{~Hz}\right), 134.9\left(4 \mathrm{CF}_{3} \mathrm{Ph}\right.$ 
$\mathrm{C}-1), 138.1(\mathrm{NPh} \mathrm{C}-1), 152.7\left(\mathrm{C}-3,{ }^{3} J(\mathrm{C} 3, \mathrm{H} 5)=7.4 \mathrm{~Hz},{ }^{3} J(\mathrm{C} 3, \mathrm{CHO})=2.0 \mathrm{~Hz}\right), 184.1\left(\mathrm{CHO},{ }^{1} J=\right.$ 175.8.0 Hz, $\left.{ }^{3} J(\mathrm{CHO}, \mathrm{H} 5)=1.2 \mathrm{~Hz}\right) .{ }^{15} \mathrm{~N}$ NMR $\left(50 \mathrm{MHz}, \mathrm{CDCl}_{3}\right): \delta_{\mathrm{N}}-159.5(\mathrm{~N}-1)$, N-2 was not found; ${ }^{19} \mathrm{~F}$ NMR $\left(470 \mathrm{MHz}, \mathrm{CDCl}_{3}\right): \delta_{\mathrm{F}}-62.7\left(\mathrm{CF}_{3}\right)$; $\mathrm{MS} m / z(\%): 317\left([\mathrm{M}+\mathrm{H}]^{+}, 100\right)$. Anal. Calcd for $\mathrm{C}_{17} \mathrm{H}_{11} \mathrm{~F}_{3} \mathrm{~N}_{2} \mathrm{O} \bullet 0.3 \mathrm{H}_{2} \mathrm{O}$ : C, 63.47; $\mathrm{H}, 3.63 ; \mathrm{N}, 8.71$. Found: $\mathrm{C}, 63.59 ; \mathrm{H}, 3.55 ; \mathrm{N}, 8.82$.

1-Phenyl-3-(3-thienyl)-1H-pyrazole-4-carbaldehyde (13). Brown solid, yield 80\%, $102 \mathrm{mg}$, mp $120^{\circ} \mathrm{C}$; IR $\left(v_{\max }, \mathrm{cm}^{-1}\right): 3126\left(\mathrm{CH}_{\text {arom }}\right), 1671(\mathrm{C}=\mathrm{O}), 756,688(\mathrm{CH}=\mathrm{CH}$ of monosubstituted benzene). ${ }^{1} \mathrm{H}$ NMR $\left(300 \mathrm{MHz}, \mathrm{CDCl}_{3}\right): \delta_{\mathrm{H}} 7.40(\mathrm{~m}, 1 \mathrm{H}, \mathrm{Ph} \mathrm{H}-4), 7.43\left(\mathrm{dd},{ }^{3} J(\mathrm{H} 5, \mathrm{H} 4)=5.0 \mathrm{~Hz}\right.$, ${ }^{4} J(\mathrm{H} 5, \mathrm{H} 2)=3.0$, Th H-5), $7.52(\mathrm{~m}, 2 \mathrm{H}, \mathrm{Ph} \mathrm{H}-3,5), 7.71\left(\mathrm{dd},{ }^{3} J(\mathrm{H} 4, \mathrm{H} 5)=5.0 \mathrm{~Hz},{ }^{4} J(\mathrm{H} 4, \mathrm{H} 2)=1.2\right.$ $\mathrm{Hz}, 1 \mathrm{H}$, Th H-4), 7.79 (m, 2H, Ph 2,6-H), 8.19 (dd, ${ }^{4} J(\mathrm{H} 2, \mathrm{H} 5)=3.0 \mathrm{~Hz},{ }^{4} J(\mathrm{H} 2, \mathrm{H} 4)=1.2,1 \mathrm{H}$, Th $\mathrm{H}-2), 8.50$ (s, 1H, H-5), 10.10 (s, 1H, CHO). ${ }^{13} \mathrm{C}$ NMR $\left(75 \mathrm{MHz}, \mathrm{CDCl}_{3}\right): \delta_{\mathrm{C}} 119.7(\mathrm{Ph} \mathrm{C}-2,6)$, $122.6\left(\mathrm{C}-4,{ }^{2} J(\mathrm{C} 4, \mathrm{CHO})=25.7 \mathrm{~Hz},{ }^{3} J(\mathrm{C} 4, \mathrm{H} 5)=7.0 \mathrm{~Hz}\right), 125.9\left(\mathrm{Th} \mathrm{C}-5,{ }^{1} J=187.7 \mathrm{~Hz}\right.$, $\left.{ }^{2} J(\mathrm{C} 5, \mathrm{H} 4)=6.0 \mathrm{~Hz},{ }^{3} J(\mathrm{C} 5, \mathrm{H} 2)=6.0 \mathrm{~Hz}\right), 126.1\left(\mathrm{Th} \mathrm{C}-2,{ }^{1} J=187.1 \mathrm{~Hz},{ }^{3} J(\mathrm{C} 2, \mathrm{H} 4)=8.7 \mathrm{~Hz},{ }^{3} J\right.$ $(\mathrm{C} 2, \mathrm{H} 5)=4.6 \mathrm{~Hz}), 127.6$ (Th C-4, $\left.{ }^{1} J=167.8 \mathrm{~Hz}\right), 127.9$ (Ph C-4), 129.7 (Ph C-3,5), 132.4 (Th C$\left.3,{ }^{2} J(\mathrm{C} 3, \mathrm{H} 2)=3.4 \mathrm{~Hz},{ }^{2} J(\mathrm{C} 3, \mathrm{H} 4)=4.6 \mathrm{~Hz},{ }^{3} \mathrm{~J}(\mathrm{C} 3, \mathrm{H} 5)=10.4 \mathrm{~Hz}\right), 132.7\left(\mathrm{C}-5,{ }^{1} J=189.3\right.$, $\left.{ }^{3} J(\mathrm{C} 5, \mathrm{CHO})=3.2\right), 138.9(\mathrm{Ph} \mathrm{C}-1), 149.3\left(\mathrm{C}-3,{ }^{3} J(\mathrm{C} 3, \mathrm{H} 5)=7.4 \mathrm{~Hz},{ }^{3} J(\mathrm{C} 3, \mathrm{ThH} 2)=3.1 \mathrm{~Hz}\right.$, $\left.{ }^{3} J(\mathrm{C} 3, \mathrm{CHO})=3.2 \mathrm{~Hz}\right), 184.0\left(\mathrm{CHO},{ }^{1} J=174.7 \mathrm{~Hz},{ }^{3} J(\mathrm{CHO}, \mathrm{H} 5)=1.4 \mathrm{~Hz}\right) ; \mathrm{MS} \mathrm{m} / z(\%): 255$ $\left([\mathrm{M}+\mathrm{H}]^{+}, 100\right)$. Anal. Calcd for $\mathrm{C}_{14} \mathrm{H}_{10} \mathrm{~N}_{2} \mathrm{OS}: \mathrm{C}, 66.12 ; \mathrm{H}, 3.96 ; \mathrm{N}, 11.02$. Found: $\mathrm{C}, 66.43 ; \mathrm{H}$, $3.97 ; \mathrm{N}, 11.34$.

3-(2-Naphthyl)-1-phenyl-1H-pyrazole-4-carbaldehyde (14). White solid, yield 79\%, $118 \mathrm{mg}$, mp 139-140 ${ }^{\circ} \mathrm{C}$ (lit. mp 140-141 $\left.{ }^{\circ} \mathrm{C}\right) .{ }^{26} \mathrm{IR}\left(v_{\max }, \mathrm{cm}^{-1}\right): 3125\left(\mathrm{CH}_{\text {arom }}\right), 1697,1668(\mathrm{C}=\mathrm{O}), 1531$, $1512(\mathrm{C}=\mathrm{C}, \mathrm{C}-\mathrm{N}), 752,692\left(\mathrm{CH}=\mathrm{CH}\right.$ of benzenes). ${ }^{1} \mathrm{H} \mathrm{NMR}\left(300 \mathrm{MHz}, \mathrm{CDCl}_{3}\right): \delta_{\mathrm{H}} 7.41(\mathrm{~m}$, 1H, Ph H-4), 7.53 (m, 2H, Ph H-3,5), 7.55 (m, 1H, Nph H-7), 7.55 (m, 1H, Nph H-6), 7.83 (m, 2H, Ph H-2,6), 7.91 (m, 1H, Nph H-5), 7.95 (m, 1H, Nph H-8), 7.98 (s, 1H, Nph H-3), 7.98 (m, 1H, Nph H-4), 8.34 (s, 1H, Nph H-1), 8.58 (s, 1H, H-5), 10.15 (s, 1H, CHO). ${ }^{13} \mathrm{C}$ NMR (125 $\left.\mathrm{MHz}, \mathrm{CDCl}_{3}\right): \delta_{\mathrm{C}} 119.8(\mathrm{Ph} \mathrm{C}-2,6), 122.7\left(\mathrm{C}-4,{ }^{2} J(\mathrm{C} 4, \mathrm{H} 5)=6.9 \mathrm{~Hz},{ }^{2} J(\mathrm{C} 4, \mathrm{CHO})=25.5 \mathrm{~Hz}\right), 126.2$ (Nph C-3), 126.5 (Nph C-7), 126.8 (Nph C-6), 127.7 (Nph C-5), 128.0 (Ph C-4), 128.5 (Nph C4), 128.5 (Nph C-8), 128.6 (Nph C-1), 128.7 (Nph C-2), 129.7 (Ph C-3,5), 131.2 (C-5, ${ }^{1} J=$ 190.1, $\left.{ }^{3} J(\mathrm{C} 5, \mathrm{CHO})=4.5\right), 133.2$ (Nph C-8a), 133.6 (Nph C-4a), 139.0 (Ph C-1), 154.7 (C-3), $185.2\left(\mathrm{CHO},{ }^{1} J=175.8,{ }^{3} J(\mathrm{CHO}, \mathrm{C} 5)=1.1 \mathrm{~Hz}\right) .{ }^{15} \mathrm{~N} \mathrm{NMR}\left(50 \mathrm{MHz}, \mathrm{CDCl}_{3}\right): \delta_{\mathrm{N}}-160.2(\mathrm{~N}-1), \mathrm{N}-$ 2 was not found; MS m/z (\%): $299\left([\mathrm{M}+\mathrm{H}]^{+}, 100\right)$. Anal. Calcd for $\mathrm{C}_{20} \mathrm{H}_{14} \mathrm{~N}_{2} \mathrm{O}$ : C, 80.52; $\mathrm{H}$, 4.73; N, 9.39. Found: C, 80.38; H, 4.76; N, 8.99.

1-(1,3-Diphenyl-1H-pyrazol-4-yl)ethanone (15). White solid, yield 81\%, $106 \mathrm{mg}, 81 \%$, mp 103-104 ${ }^{\circ} \mathrm{C}$ (lit. mp 99-104 $\left.{ }^{\circ} \mathrm{C}\right) ;{ }^{29} \mathrm{IR}\left(v_{\max }, \mathrm{cm}^{-1}\right): 3119\left(\mathrm{CH}_{\text {arom }}\right), 1665(\mathrm{C}=\mathrm{O}), 1525,1448(\mathrm{C}=\mathrm{C}$, $\mathrm{C}-\mathrm{N}), 754,732,696,688\left(\mathrm{CH}=\mathrm{CH}\right.$ of monosubstituted benzenes). ${ }^{1} \mathrm{H} \mathrm{NMR}\left(300 \mathrm{MHz}, \mathrm{CDCl}_{3}\right)$ : $\delta_{\mathrm{H}} 2.39\left(\mathrm{~s}, 3 \mathrm{H}, \mathrm{CH}_{3}\right), 7.39$ (m, 1H, NPh H-4), 7.45 (m, 1H, CPh H-4), 7.46 (m, 2H, CPh H-3,5), 7.50 (m, 2H, NPh H-3,5), 7.75 (m, 2H, CPh H-2,6), 7.78 (m, 2H, NPh H-2,6), 8.46 (s, 1H, H-5). ${ }^{13} \mathrm{C} \mathrm{NMR}\left(75 \mathrm{MHz}, \mathrm{CDCl}_{3}\right): \delta_{\mathrm{C}} 29.3\left(\mathrm{CH}_{3},{ }^{1} J=127.6 \mathrm{~Hz}\right), 119.6(\mathrm{NPh} \mathrm{C}-2,6), 122.9(\mathrm{C}-4$, $\left.{ }^{2} J(\mathrm{C} 4, \mathrm{C} 5)=7.3 \mathrm{~Hz},{ }^{3} J\left(\mathrm{C} 4, \mathrm{CH}_{3}\right)=1.1 \mathrm{~Hz}\right), 127.6(\mathrm{NPh} \mathrm{C}-4), 128.1$ (CPh C-3,5), 128.9 (CPh C-4), 129.4 (CPh C-2,6), 129.6 (NPh C-3,5), 131.5 (C-5, $\left.{ }^{1} J=188.0\right), 132.1$ (CPh C-1), 139.2 (NPh C1), $153.5\left(\mathrm{C}-3,{ }^{3} J(\mathrm{C} 3, \mathrm{H} 5)=7.5 \mathrm{~Hz},{ }^{3} J(\mathrm{C} 3, \mathrm{CPhH}-2,6)=3.9 \mathrm{~Hz}\right), 192.4\left(\mathrm{CO},{ }^{2} J\left(\mathrm{CO}, \mathrm{CH}_{3}\right)=7.3 \mathrm{~Hz}\right)$; 
MS $m / z(\%): 263\left([\mathrm{M}+\mathrm{H}]^{+}, 100\right)$. Anal. Calcd for $\mathrm{C}_{17} \mathrm{H}_{14} \mathrm{~N}_{2} \mathrm{O} \cdot 0.3 \mathrm{H}_{2} \mathrm{O}: \mathrm{C}, 76.27 ; \mathrm{H}, 5.50 ; \mathrm{N}$, 10.46. Found: C, 76.03; H, 5.32; N, 10.10.

1-[3-(3-Chlorophenyl)-1-phenyl-1H-pyrazol-4-yl]ethanone (16). Yellow solid, yield 50\%, 74 $\mathrm{mg}, \mathrm{mp} 76-77{ }^{\circ} \mathrm{C}$; IR $\left(v_{\max }, \mathrm{cm}^{-1}\right): 3130\left(\mathrm{CH}_{\text {arom }}\right), 1674(\mathrm{C}=\mathrm{O}), 1527,1504,1450(\mathrm{C}=\mathrm{C}, \mathrm{C}-\mathrm{N})$, 797, 760, $688\left(\mathrm{CH}=\mathrm{CH}\right.$ of mono- and disubstituted benzenes). ${ }^{1} \mathrm{H}$ NMR $\left(300 \mathrm{MHz}, \mathrm{CDCl}_{3}\right): \delta_{\mathrm{H}}$ 2.45 (s, 3H, $\left.\mathrm{CH}_{3}\right), 7.37$ (m, 1H, 3ClPh H-5), 7.38 (m, 1H, NPh H-4), 7.40 (m, 1H, 3ClPh H-4), 7.51 (m, 2H, NPh H-3,5), 7.70 (m, 1H, 3ClPh H-6), 7.77 (m, 2H, NPh H-2,6), 7.82 (m, 1H, 3ClPh H-2), 8.45 (s, 1H, H-5). ${ }^{13} \mathrm{C}$ NMR (75 MHz, $\left.\mathrm{CDCl}_{3}\right): \delta_{\mathrm{C}} 29.3\left(\mathrm{CH}_{3},{ }^{1} \mathrm{~J}=127.6 \mathrm{~Hz}\right), 119.6$ $(\mathrm{NPh} \mathrm{C}-2,6), 122.6\left(\mathrm{C}-4,{ }^{2} J(\mathrm{C} 4, \mathrm{C} 5)=7.3 \mathrm{~Hz},{ }^{3} \mathrm{~J}(\mathrm{C} 4, \mathrm{CH} 3)=1.1 \mathrm{~Hz}\right), 127.7$ (3ClPh C-6), 127.8 (NPh C-4), 128.9 (3ClPh C-4), 129.2 (3ClPh C-5), 129.4 (3ClPh C-2), 129.6 (NPh C-3,5), 131.8 $\left(\mathrm{C}-5,{ }^{1} J=188.0\right), 134.0$ (3ClPh C-3), 134.2 (3ClPh C-1), 139.0 (NPh C-1), 152.0 (C-3, $\left.{ }^{3} J(\mathrm{C} 3, \mathrm{H} 5)=7.6 \mathrm{~Hz}\right), 191.8\left(\mathrm{CO},{ }^{2} J(\mathrm{CO}, \mathrm{CH} 3)=6.0 \mathrm{~Hz}\right) .{ }^{15} \mathrm{~N} \mathrm{NMR}\left(50 \mathrm{MHz}, \mathrm{CDCl}_{3}\right): \delta_{\mathrm{N}}-163.3$ $(\mathrm{N}-1)$, N-2 was not found; $\mathrm{MS} m / z(\%)$ : 321/319 ([M+Na $\left.]^{+}, 30 / 100\right)$. Anal. Calcd for $\mathrm{C}_{17} \mathrm{H}_{13} \mathrm{ClN}_{2} \mathrm{O}: \mathrm{C}, 68.81 ; \mathrm{H}, 4.42 ; \mathrm{N}, 9.44$. Found: C, 68.99; H, 4.46; N, 9.80.

1-\{3-[4-(Trifluoromethyl)phenyl-1-phenyl-]-1H-pyrazol-4-yl\}ethanone (17). White solid, yield 82\%, $136 \mathrm{mg}, \mathrm{mp} 73-75^{\circ} \mathrm{C}$; IR $\left(v_{\max }, \mathrm{cm}^{-1}\right): 3136\left(\mathrm{CH}_{\text {arom }}\right), 1668(\mathrm{C}=\mathrm{O}), 1524(\mathrm{C}=\mathrm{C}, \mathrm{C}-$ $\mathrm{N}), 1327,1126,1068(\mathrm{C}-\mathrm{F}), 758,686\left(\mathrm{CH}=\mathrm{CH}\right.$ of mono- and disubstituted benzenes). ${ }^{1} \mathrm{H}$ NMR $\left(500 \mathrm{MHz}, \mathrm{CDCl}_{3}\right): \delta_{\mathrm{H}} 2.49$ (s, 3H, CH$\left.)_{3}\right), 7.40$ (m, 2H, NPh H-4), 7.52 (m, 2H, NPh H-3,5), 7.70 (m, 2H, 4CF 3 Ph H-3,5), 7.78 (m, 2H, NPh H-2,6), 7.94 (m, 2H, 4CF 3 Ph H-2,6), 8.47 (s, 1H, H5). ${ }^{13} \mathrm{C}$ NMR $\left(125 \mathrm{MHz}, \mathrm{CDCl}_{3}\right): \delta_{\mathrm{C}} 29.2\left(\mathrm{CH}_{3},{ }^{1} \mathrm{~J}=127.6 \mathrm{~Hz}\right), 119.6(\mathrm{NPh} \mathrm{C}-2,6), 122.5(\mathrm{C}-4$, $\left.{ }^{2} J(\mathrm{C} 4, \mathrm{C} 5)=7.4 \mathrm{~Hz},{ }^{3} J\left(\mathrm{C} 4, \mathrm{CH}_{3}\right)=1.0 \mathrm{~Hz}\right), 124.2\left(\mathrm{CF}_{3},{ }^{1} J=272.3 \mathrm{~Hz}\right), 124.9\left(4 \mathrm{CF}_{3} \mathrm{Ph} \mathrm{C}-3,5\right)$, 127.9 (NPh C-4), 129.67 (NPh C-3,5), 129.73 (4CF 3 Ph C-2,6), 130.6 (4CF 3 Ph C-4), 132.0 (C-5, $\left.{ }^{1} J=187.9\right), 135.9\left(4 \mathrm{CF}_{3} \mathrm{Ph} \mathrm{C}-1\right), 139.0(\mathrm{NPh} \mathrm{C}-1), 152.0\left(\mathrm{C}-3,{ }^{3} J(\mathrm{C} 3, \mathrm{H} 5)=7.6 \mathrm{~Hz},{ }^{3} J(\mathrm{C} 3\right.$, $\left.\left.4 \mathrm{CF}_{3} \mathrm{Ph} \mathrm{C}-2,6\right)=3.8 \mathrm{~Hz}\right), 191.7\left(\mathrm{CO},{ }^{2} J\left(\mathrm{CO}, \mathrm{CH}_{3}\right)=6.0 \mathrm{~Hz}\right) .{ }^{19} \mathrm{~F}$ NMR $\left(470 \mathrm{MHz}, \mathrm{CDCl}_{3}\right): \delta_{\mathrm{F}}$ $-62.6\left(\mathrm{CF}_{3}\right)$; $\mathrm{MS} m / z(\%): 331\left([\mathrm{M}+\mathrm{H}]^{+}, 100\right)$. Anal. Calcd for $\mathrm{C}_{18} \mathrm{H}_{13} \mathrm{~F}_{3} \mathrm{~N}_{2} \mathrm{O}: \mathrm{C}, 65.45 ; \mathrm{H}, 3.97$; N, 8.48. Found: C, 65.24; H, 4.06; N, 8.32.

1-[1-Phenyl-3-(3-thienyl)-1H-pyrazol-4-yl]ethanone (18). White solid, yield 60\%, $80 \mathrm{mg}$, 60\%, mp 80-81 ${ }^{\circ} \mathrm{C}$; IR ( $\left.v_{\max }, \mathrm{cm}^{-1}\right): 3117\left(\mathrm{CH}_{\text {arom }}\right), 1675(\mathrm{C}=\mathrm{O}), 1519,1499(\mathrm{C}=\mathrm{C}, \mathrm{C}-\mathrm{N}), 771$, $691\left(\mathrm{CH}=\mathrm{CH}\right.$ of monosubstituted benzene). ${ }^{1} \mathrm{H} \mathrm{NMR}\left(300 \mathrm{MHz}, \mathrm{CDCl}_{3}\right): \delta_{\mathrm{H}} 2.51\left(\mathrm{~s}, 3 \mathrm{H}, \mathrm{CH}_{3}\right)$, $7.35\left(\mathrm{dd},{ }^{3} J(\mathrm{H} 5, \mathrm{H} 4)=5.0 \mathrm{~Hz},{ }^{4} J(\mathrm{H} 5, \mathrm{H} 2)=3.1\right.$, Th H-5), 7.37 (m, 1H, Ph H-4), 7.51 (m, 2H, Ph H3,5), $7.72\left(\mathrm{dd},{ }^{4} J(\mathrm{H} 4, \mathrm{H} 2)=1.2 \mathrm{~Hz}, 1 \mathrm{H}, \mathrm{Th} \mathrm{H}-4\right), 7.78$ (m, 2H, Ph 2,6-H), 8.34 (dd, 1H, Th H-2), 8.42 (s, 1H, H-5). ${ }^{13} \mathrm{C}$ NMR $\left(75 \mathrm{MHz}, \mathrm{CDCl}_{3}\right): \delta_{\mathrm{C}} 29.3\left(\mathrm{CH}_{3},{ }^{1} J=127.6 \mathrm{~Hz}\right), 119.6(\mathrm{Ph} \mathrm{C}-2,6)$, $122.2\left(\mathrm{C}-4,{ }^{2} J(\mathrm{C} 4, \mathrm{C} 5)=7.2 \mathrm{~Hz}\right), 124.7\left(\mathrm{Th} \mathrm{C}-5,{ }^{1} \mathrm{~J}=185.8 \mathrm{~Hz},{ }^{2} J(\mathrm{C} 5, \mathrm{H} 4)=7.8 \mathrm{~Hz},{ }^{3} J(\mathrm{C} 5, \mathrm{H} 2)=6.0\right.$ $\mathrm{Hz}), 126.6$ (Th C-2, $\left.{ }^{1} J=188.5 \mathrm{~Hz},{ }^{3} J(\mathrm{C} 2, \mathrm{H} 4)=8.4 \mathrm{~Hz},{ }^{3} J(\mathrm{C} 2, \mathrm{H} 5)=4.5 \mathrm{~Hz}\right), 128.4\left(\mathrm{Th} \mathrm{C}-4,{ }^{1} J=\right.$ $\left.169.9 \mathrm{~Hz},{ }^{2} J(\mathrm{C} 4, \mathrm{H} 5)=5.1 \mathrm{~Hz},{ }^{3} J(\mathrm{C} 4, \mathrm{H} 2)=8.7 \mathrm{~Hz}\right), 127.6(\mathrm{Ph} \mathrm{C}-4), 129.6(\mathrm{Ph} \mathrm{C}-3,5), 132.8(\mathrm{Th}$ $\left.\mathrm{C}-3,{ }^{2} J(\mathrm{C} 3, \mathrm{H} 2)=3.1 \mathrm{~Hz},{ }^{2} J(\mathrm{C} 3, \mathrm{H} 4)=4.1 \mathrm{~Hz},{ }^{3} J(\mathrm{C} 3, \mathrm{H} 5)=10.0 \mathrm{~Hz}\right), 132.0\left(\mathrm{C}-5,{ }^{1} J=187.1\right), 139.1$ $(\mathrm{Ph} \mathrm{C}-1), 148.6\left(\mathrm{C}-3,{ }^{3} J(\mathrm{C} 3, \mathrm{H} 5)=7.6 \mathrm{~Hz},{ }^{3} J(\mathrm{C} 3, \mathrm{ThH} 2)=3.0 \mathrm{~Hz},{ }^{3} J(\mathrm{C} 3, \mathrm{ThH} 4)=1.3 \mathrm{~Hz}\right), 191.8$ $\left(\mathrm{CO},{ }^{1} J\left(\mathrm{CO}, \mathrm{CH}_{3}\right)=5.8 \mathrm{~Hz}\right) .{ }^{15} \mathrm{~N}$ NMR $\left(50 \mathrm{MHz}, \mathrm{CDCl}_{3}\right): \delta_{\mathrm{N}}-165.2(\mathrm{~N}-1), \mathrm{N}-2$ was not found; MS $m / z(\%): 269\left([\mathrm{M}+\mathrm{H}]^{+}, 100\right)$. Anal. Calcd for $\mathrm{C}_{15} \mathrm{H}_{12} \mathrm{~N}_{2} \mathrm{OS} \bullet 0.5 \mathrm{H}_{2} \mathrm{O}: \mathrm{C}, 64.96 ; \mathrm{H}, 4.72 ; \mathrm{N}$, 10.10. Found: C, 64.97; H, 4.57; N, 9.92. 
1-[3-(2-Naphtyl)-1-phenyl-1H-pyrazol-4-yl]ethanone (19). White solid, yield 77\%, $120 \mathrm{mg}$, mp 114-116 ${ }^{\circ} \mathrm{C}$; IR $\left(v_{\max }, \mathrm{cm}^{-1}\right): 3130\left(\mathrm{CH}_{\text {arom }}\right), 1670(\mathrm{C}=\mathrm{O}), 1527,1502(\mathrm{C}=\mathrm{C}, \mathrm{C}-\mathrm{N}), 758,688$ $\left(\mathrm{CH}=\mathrm{CH}\right.$ of benzenes). ${ }^{1} \mathrm{H}$ NMR $\left(500 \mathrm{MHz}, \mathrm{CDCl}_{3}\right): \delta_{\mathrm{H}} 2.43\left(\mathrm{~s}, 3 \mathrm{H}, \mathrm{CH}_{3}\right), 7.38(\mathrm{~m}, 1 \mathrm{H}, \mathrm{Ph} \mathrm{H}-4)$, 7.51 (m, 2H, Ph H-3,5), 7.51 (m, 1H, Nph H-7), 7.52 (m, 1H, Nph H-6), 7.81 (m, 2H, Ph H-2,6), $7.87\left(\mathrm{~d},{ }^{3} J(\mathrm{H} 3, \mathrm{H} 4)=8.6 \mathrm{~Hz}, 1 \mathrm{H}, \mathrm{Nph} \mathrm{H}-3\right), 7.89$ (m, 1H, Nph H-5), 7.92 (m, 1H, Nph H-4), 7.93 (m, 1H, Nph H-8), 8.31 (s, 1H, Nph H-1), 8.49 (s, 1H, H-5). ${ }^{13} \mathrm{C}$ NMR $\left(125 \mathrm{MHz}, \mathrm{CDCl}_{3}\right): \delta_{\mathrm{C}}$ $29.4\left(\mathrm{CH}_{3},{ }^{1} J\left(\mathrm{CH}_{3}\right)=127.6 \mathrm{~Hz}\right), \quad 119.6 \quad(\mathrm{Ph} \quad \mathrm{C}-2,6), \quad 123.0 \quad\left(\mathrm{C}-4, \quad{ }^{2} J(\mathrm{C} 4, \mathrm{H} 5)=7.3 \quad \mathrm{~Hz}\right.$, $\left.{ }^{3} J\left(\mathrm{C} 4, \mathrm{CH}_{3}\right)=1.2 \mathrm{~Hz}\right), 126.1$ (Nph C-7), 126.5 (Nph C-6), 127.0 (Nph C-3), 127.6 (Nph C-4), 127.64 (Ph C-4), 127.67 (Nph C-5), 128.5 (Nph C-8), 128.8 (Nph C-1), 129.6 (Ph C-3,5), 129.9 (Nph C-2), 131.7 (C-5, $\left.{ }^{1} J=188.1 \mathrm{~Hz}\right), 133.1$ (Nph C-8a), 133.4 (Nph C-4a), 139.1 (Ph C-1), $153.5\left(\mathrm{C}-3,{ }^{3} J(\mathrm{C} 3, \mathrm{H} 5)=7.5 \mathrm{~Hz}\right), 192.4\left(\mathrm{CO},{ }^{2} J\left(\mathrm{CO}_{2} \mathrm{CH}_{3}\right)=6.0 \mathrm{~Hz}\right) .{ }^{15} \mathrm{~N} \mathrm{NMR}\left(50 \mathrm{MHz}, \mathrm{CDCl}_{3}\right)$ : $\delta_{\mathrm{N}}-163.5(\mathrm{~N}-1), \mathrm{N}-2$ was not found; $\mathrm{MS} m / z(\%): 335\left([\mathrm{M}+\mathrm{Na}]^{+}, 100\right)$. Anal. Calcd for $\mathrm{C}_{21} \mathrm{H}_{16} \mathrm{~N}_{2} \mathrm{O} \bullet 0.2 \mathrm{H}_{2} \mathrm{O}$ : C, 79.83; H, 5.23; N, 8.87. Found: C, 79.88; H, 5.21; N, 8.90.

General procedure for the preparation of 4-substituted 1-phenyl-1H-pyrazol-4carbaldehydes and ketones by Sonogashira cross-coupling reaction: method $B$

To a solution of appropriate pyrazole 7 or $9(0.5 \mathrm{mmol})$ in dry DMF $(5 \mathrm{~mL})$ under argon atmosphere triethylamine $(0.11 \mathrm{~mL}, 0.75 \mathrm{mmol})$, phenylacetylene $(0.08 \mathrm{~mL}, 0.75 \mathrm{mmol})$, $\mathrm{Pd}\left(\mathrm{PPh}_{3}\right)_{2} \mathrm{Cl}_{2}(35 \mathrm{mg}, 0.05 \mathrm{mmol})$ and $\mathrm{CuI}(9 \mathrm{mg}, 0.05 \mathrm{mmol})$ were added. The mixture was stirred for the given time under argon atmosphere at $65^{\circ} \mathrm{C}$, diluted with water and the extraction was done with ethyl acetate. The combined organic layers were washed with brine, dried over $\mathrm{Na}_{2} \mathrm{SO}_{4}$, the solvent was evaporated. The residue was purified by flash chromatography $\left(\mathrm{SiO}_{2}\right.$, eluent: ethyl acetate/n-hexane, 1:5, v/v) to yield compounds 20, 21.

1-Phenyl-3-(phenylethynyl)-1H-pyrazole-4-carbaldehyde (20). Pale solid, yield 81\%, $110 \mathrm{mg}$, 81\%, mp 134-136 ${ }^{\circ} \mathrm{C}$; IR ( $\left.v_{\max }, \mathrm{cm}^{-1}\right): 3119\left(\mathrm{CH}_{\text {arom }}\right), 1675(\mathrm{C}=\mathrm{O}), 1527,1504(\mathrm{C}=\mathrm{C}, \mathrm{C}-\mathrm{N}), 753$, $685\left(\mathrm{CH}=\mathrm{CH}\right.$ of monosubstituted benzenes). ${ }^{1} \mathrm{H}$ NMR $\left(300 \mathrm{MHz}, \mathrm{CDCl}_{3}\right): \delta_{\mathrm{H}} 7.40(\mathrm{~m}, 1 \mathrm{H}, \mathrm{NPh}$ $\mathrm{H}-4), 7.40$ (m, 3H, CPh H-3,4,5), 7.50 (m, 2H, NPh H-3,5), 7.62 (m, 2H, CPh H-2,6), 7.74 (m, 2H, NPh H-2,6), 8.44 (s, 1H, H-5), 10.10 (s, 1H, CHO). ${ }^{13} \mathrm{C}$ NMR (75 MHz, $\left.\mathrm{CDCl}_{3}\right): \delta_{\mathrm{C}} 78.8$ $(\mathrm{PhC} \equiv C), 94.9\left(\mathrm{PhC} \equiv \mathrm{C},{ }^{3} J(\mathrm{C}, \mathrm{CPh} 2,6-\mathrm{H})=5.4 \mathrm{~Hz}\right), 119.8(\mathrm{NPh} \mathrm{C}-2,6), 121.7$ (CPh C-1), 125.8 $\left(\mathrm{C}-4,{ }^{2} J(\mathrm{C} 4, \mathrm{CHO})=25.7 \mathrm{~Hz},{ }^{2} J(\mathrm{C} 4, \mathrm{C} 5)=7.0 \mathrm{~Hz}\right), 128.3(\mathrm{NPh} \mathrm{C}-4), 128.5(\mathrm{CPh} \mathrm{C}-3,5), 129.3$ (CPh C-4), 129.7 (NPh C-3,5), 128.6 (C-5, $\left.{ }^{1} \mathrm{~J}=192.4,{ }^{3} \mathrm{~J}(\mathrm{C} 5, \mathrm{CHO})=4.9 \mathrm{~Hz}\right), 132.0(\mathrm{CPh} \mathrm{C}-2,6)$, $138.5\left(\mathrm{C}-3,{ }^{3} J(\mathrm{C} 3, \mathrm{H} 5)=8.4 \mathrm{~Hz}\right), 138.7(\mathrm{NPh} \mathrm{C}-1), 184.3\left(\mathrm{CHO},{ }^{1} J=177.9 \mathrm{~Hz}\right) ; \mathrm{MS} \mathrm{m} / z(\%): 295$ $\left([\mathrm{M}+\mathrm{Na}]^{+}\right.$, 100). Anal. Calcd for $\mathrm{C}_{18} \mathrm{H}_{12} \mathrm{~N}_{2} \mathrm{O} \bullet 0.6 \mathrm{H}_{2} \mathrm{O}: \mathrm{C}, 76.36 ; \mathrm{H}, 4.70 ; \mathrm{N}, 9.89$. Found: C, 76.43; H, 4.52; N, 9.49.

1-[1-Phenyl-3-(2-phenylethynyl)-1H-pyrazol-4-yl]ethanone (21). Pale solid, yield 65\%, 93 $\mathrm{mg}, \mathrm{mp} 112-113{ }^{\circ} \mathrm{C}$; IR ( $\left.v_{\max }, \mathrm{cm}^{-1}\right): 3129\left(\mathrm{CH}_{\text {arom }}\right), 1668(\mathrm{C}=\mathrm{O}), 1523,1498(\mathrm{C}=\mathrm{C}, \mathrm{C}-\mathrm{N}), 767$, 751, 706, $691\left(\mathrm{CH}=\mathrm{CH}\right.$ of monosubstituted benzenes). ${ }^{1} \mathrm{H} \mathrm{NMR}\left(300 \mathrm{MHz}, \mathrm{CDCl}_{3}\right): \delta_{\mathrm{H}} 2.73(\mathrm{~s}$, $3 \mathrm{H}, \mathrm{CH}_{3}$ ), 7.38 (m, 1H, NPh H-4), 7.39 (m, 3H, CPh H-3,4,5), 7.49 (m, 2H, NPh H-3,5), 7.62 (m, 2H, CPh H-2,6), 7.74 (m, 2H, NPh H-2,6), 8.45 (s, 1H, H-5). ${ }^{13} \mathrm{C} \mathrm{NMR}\left(75 \mathrm{MHz}, \mathrm{CDCl}_{3}\right): \delta_{\mathrm{C}}$ $29.0\left(\mathrm{CH}_{3},{ }^{1} J=127.8 \mathrm{~Hz}\right), 81.2\left(\mathrm{PhC} \equiv C,{ }^{3} J(\mathrm{C}, \mathrm{CPh} 2,6-\mathrm{H})=5.3 \mathrm{~Hz}\right), 94.7(\mathrm{PhC} \equiv \mathrm{C}$, 
$\left.{ }^{3} J(\mathrm{C}, \mathrm{CPh} 2,6-\mathrm{H})=5.4 \mathrm{~Hz}\right), 119.7(\mathrm{NPh} \mathrm{C}-2,6), 122.0(\mathrm{CPh} \mathrm{C}-1), 127.0\left(\mathrm{C}-4,{ }^{2} J(\mathrm{C} 4, \mathrm{C} 5)=6.7 \mathrm{~Hz}\right.$, $\left.{ }^{3} J\left(\mathrm{C} 4, \mathrm{CH}_{3}\right)=1.4 \mathrm{~Hz}\right), 128.0$ (NPh C-4), 128.4 (CPh C-3,5), 129.2 (CPh C-4), 129.6 (NPh C-3,5), $129.9\left(\mathrm{C}-5,{ }^{1} J=191.5\right), 131.7(\mathrm{CPh} \mathrm{C}-2,6), 138.8(\mathrm{NPh} \mathrm{C}-1), 136.0\left(\mathrm{C}-3,{ }^{3} J(\mathrm{C} 3, \mathrm{H} 5)=8.1 \mathrm{~Hz}\right)$, $192.1\left(\mathrm{CO},{ }^{2} J\left(\mathrm{CO}, \mathrm{CH}_{3}\right)=6.2 \mathrm{~Hz}\right) ; \mathrm{MS} m / z(\%): 287\left([\mathrm{M}+\mathrm{H}]^{+}, 100\right)$. Anal. Calcd for $\mathrm{C}_{19} \mathrm{H}_{14} \mathrm{~N}_{2} \mathrm{O} \bullet 0.4 \mathrm{H}_{2} \mathrm{O}: \mathrm{C}, 77.74 ; \mathrm{H}, 5.08 ; \mathrm{N}, 9.54$. Found: C, 77.82; H, 4.91; N, 9.26.

General procedure for the preparation of 4-substituted 1-phenyl-1H-pyrazol-4carbaldehydes and ketones by Heck cross-coupling reaction: $\operatorname{method} \mathbf{C}$

To a solution of appropriate pyrazole 7 or $9(0.5 \mathrm{mmol})$ in dry DMF $(5 \mathrm{~mL})$ under argon atmosphere triethylamine $(0.11 \mathrm{~mL}, 0.75 \mathrm{mmol})$, appropriate alkene $(1 \mathrm{mmol})$ and $\mathrm{Pd}\left(\mathrm{PPh}_{3}\right)_{2} \mathrm{Cl}_{2}$ (35 mg, $0.05 \mathrm{mmol}$ ) were added. The reaction mixture was stirred for the given time at $100{ }^{\circ} \mathrm{C}$ under argon atmosphere, then diluted with water and exhaustively extracted with ethyl acetate. The combined organic layers were washed with brine, dried over $\mathrm{Na}_{2} \mathrm{SO}_{4}$ and evaporated under reduced pressure. The residue was purified by flash chromatography $\left(\mathrm{SiO}_{2}\right.$, eluent: ethyl acetate/ $n$-hexane, $1: 4, \mathrm{v} / \mathrm{v}$ ) to yield compounds 22-27.

Ethyl (2E)-3-(4-formyl-1-phenyl-1H-pyrazol-3-yl)acrylate (22). Yellow liquid, yield 37\%, 50 mg; IR ( $\left.v_{\max }, \mathrm{cm}^{-1}\right): 3113\left(\mathrm{CH}_{\text {arom }}\right), 1700,1682(\mathrm{C}=\mathrm{O}), 1196(\mathrm{C}-\mathrm{O}), 751,688(\mathrm{CH}=\mathrm{CH}$ of monosubstituted benzene). ${ }^{1} \mathrm{H}$ NMR $\left(300 \mathrm{MHz}, \mathrm{CDCl}_{3}\right): \delta_{\mathrm{H}} 1.33\left(\mathrm{t}, 3 \mathrm{H},{ }^{3} \mathrm{~J}=7.3 \mathrm{~Hz}, \mathrm{CH}_{3}\right), 4.28$ (q, $\left.2 \mathrm{H},{ }^{3} \mathrm{~J}=7.3 \mathrm{~Hz}, \mathrm{CH}_{2}\right), 6.57$ (d, $\left.1 \mathrm{H},{ }^{3} J_{\text {trans }}=16.1 \mathrm{~Hz}, \mathrm{CHCHCO}\right), 7.42$ (m, 1H, Ph H-4), 7.51 (m, 2H, Ph H-3,5), 7.74 (m, 2H Ph H-2,6), 7.98 (d, 1H, $\left.{ }^{3} J_{\text {trans }}=16.1 \mathrm{~Hz}, \mathrm{CHCHCO}\right), 8.44$ (s, 1H, $\mathrm{H}-5), 10.08$ (CHO). ${ }^{13} \mathrm{C}$ NMR $\left(75 \mathrm{MHz}, \mathrm{CDCl}_{3}\right): \delta_{\mathrm{C}} 14.3\left(\mathrm{CH}_{3}\right), 60.7\left(\mathrm{CH}_{2}\right), 119.7(\mathrm{Ph} \mathrm{C}-2,6)$, 123.8 (COCH), 127.0, 128.3, 129.7 (Ph C-3,5), 130.1, 132.80 (COCHCH), 138.7 (Ph C-1), 148.5 (C-3), 166.5 (CHCHCO), $183.4(\mathrm{CHO}) ; \mathrm{MS} \mathrm{m} / \mathrm{z}(\%): 271\left([\mathrm{M}+\mathrm{H}]^{+}, 100\right)$. Anal. Calcd for $\mathrm{C}_{15} \mathrm{H}_{14} \mathrm{~N}_{2} \mathrm{O}_{3}$ : C, 66.66; H, 5.22; N, 10.36. Found: C, 66.37; H, 5.53; N, 10.58 .

tert-Butyl (2E)-3-(4-formyl-1-phenyl-1H-pyrazol-3-yl)acrylate (23). Beige liquid, yield 13\%, $20 \mathrm{mg}$. IR ( $\left.v_{\max }, \mathrm{cm}^{-1}\right): 3122\left(\mathrm{CH}_{\text {arom }}\right), 2978\left(\mathrm{CH}_{\text {aliph }}\right), 1710,1687(\mathrm{C}=\mathrm{O}), 757,689(\mathrm{CH}=\mathrm{CH}$ of monosubstituted benzenes). ${ }^{1} \mathrm{H}$ NMR $\left(300 \mathrm{MHz}, \mathrm{CDCl}_{3}\right): \delta_{\mathrm{H}} 1.54\left(\mathrm{~s}, 9 \mathrm{H}, \mathrm{CH}_{3}\right), 6.96(\mathrm{~d}, 1 \mathrm{H}$, $\left.{ }^{3} J_{\text {trans }}=15.75 \mathrm{~Hz}, \mathrm{COCHCH}\right), 7.40$ (m, 1H, Ph H-4), 7.52 (m, 2H, Ph H-3,5), 7.75 (m, 2H, Ph H2,6), $7.90\left(\mathrm{~d}, 1 \mathrm{H},{ }^{3} J_{\text {trans }}=15.75 \mathrm{~Hz}, \mathrm{COCHCH}\right), 8.43$ (s, 1H, H-5), 10.09 (CHO); MS m/z (\%): $339\left([\mathrm{M}+\mathrm{K}+\mathrm{H}]^{+}\right.$, 100). Anal. Calcd for $\mathrm{C}_{17} \mathrm{H}_{18} \mathrm{~N}_{2} \mathrm{O}_{3}$ : C, 68.44; H, 6.08; N, 9.39. Found: C, 68.80; H, 6.17; N, 9.64.

1-Phenyl-3-[(E)-2-phenylethenyl]-1H-pyrazole-4-carbaldehyde (24). Yellow liquid, yield 15\%, $21 \mathrm{mg}$. IR $\left(v_{\max }, \mathrm{cm}^{-1}\right): 3160\left(\mathrm{CH}_{\text {arom }}\right), 1682(\mathrm{C}=\mathrm{O}), 753,687(\mathrm{CH}=\mathrm{CH}$ of monosubstituted benzenes). ${ }^{1} \mathrm{H}$ NMR (300 MHz, $\mathrm{CDCl}_{3}$ ): $\delta_{\mathrm{H}}$ 7.31-7.42 (m, 4H, Ar), 7.48-7.63 (m, 4H, Ar), 7.60 $\left(\mathrm{d}, 1 \mathrm{H},{ }^{3} J_{\text {trans }}=16.5 \mathrm{~Hz}, \mathrm{CHCHPh}\right), 7.78(\mathrm{~m}, 2 \mathrm{H}, \mathrm{NPh} \mathrm{H}-2,6), 7.75\left(\mathrm{~d}, 1 \mathrm{H},{ }^{3} J_{\text {trans }}=16.5 \mathrm{~Hz}\right.$, $\mathrm{CHCHPh}), 8.41$ (s, 1H, H-5), 10.12 (CHO); MS m/z (\%): 275 ([M+ H $\left.]^{+}, 100\right)$. Anal. Calcd for: $\mathrm{C}_{18} \mathrm{H}_{14} \mathrm{~N}_{2} \mathrm{O}$ : C, 78.81; H, 5.14; N, 10.21. Found: C, 79.10; H, 5.16; N, 9.97.

Ethyl (2E)-3-(4-acetyl-1-phenyl-1H-pyrazol-3-yl)acrylate (25). Yellow solid, yield 78\%, 111 $\mathrm{mg}, \mathrm{mp} 105-10{ }^{\circ} \mathrm{C}$; IR $\left(v_{\max }, \mathrm{cm}^{-1}\right): 3133\left(\mathrm{CH}_{\text {arom }}\right), 1702,1692,1673(\mathrm{C}=\mathrm{O}), 1596,1530(\mathrm{C}=\mathrm{C}$, $\mathrm{C}-\mathrm{N}), 1280,1252,1204(\mathrm{C}-\mathrm{O}), 767,688\left(\mathrm{CH}=\mathrm{CH}\right.$ of monosubstituted benzene). ${ }^{1} \mathrm{H}$ NMR $(300$ 
$\left.\mathrm{MHz}, \mathrm{CDCl}_{3}\right): \delta_{\mathrm{H}} 1.33\left(\mathrm{t}, 3 \mathrm{H},{ }^{3} J=7.1 \mathrm{~Hz}, \mathrm{CH}_{2} \mathrm{CH}_{3}\right), 2.52\left(\mathrm{~s}, 3 \mathrm{H}, \mathrm{COCH}_{3}\right), 4.26\left(\mathrm{q}, 2 \mathrm{H},{ }^{3} J=7.1\right.$ $\left.\mathrm{Hz}, \mathrm{CH}_{2}\right), 6.96\left(\mathrm{~d}, 1 \mathrm{H},{ }^{3} J_{\text {trans }}=16.0 \mathrm{~Hz}, \mathrm{COCHCH}\right), 7.38(\mathrm{~m}, 1 \mathrm{H}, \mathrm{Ph} \mathrm{H}-4), 7.50(\mathrm{~m}, 2 \mathrm{H}, \mathrm{Ph} \mathrm{H}-$ 3,5), 7.74 (m, 2H, Ph H-2,6), 8.19 (d, $\left.{ }^{3} J_{\text {trans }}=16.0 \mathrm{~Hz}, 1 \mathrm{H}, \mathrm{COCHCH}\right), 8.36(\mathrm{~s}, 1 \mathrm{H}, \mathrm{H}-5) .{ }^{13} \mathrm{C}$ NMR $\left(125 \mathrm{MHz}, \mathrm{CDCl}_{3}\right): \delta_{\mathrm{C}} 14.1\left(\mathrm{CH}_{2} \mathrm{CH}_{3},{ }^{1} \mathrm{~J}=127.0 \mathrm{~Hz},{ }^{2} J\left(\mathrm{CH}_{2} \mathrm{CH}_{3}, \mathrm{CH}_{2}\right)=26.0 \mathrm{~Hz}\right), 28.9$ $\left(\mathrm{COCH}_{3},{ }^{1} J=127.6 \mathrm{~Hz}\right), 60.5\left(\mathrm{CH}_{2} \mathrm{CH}_{3},{ }^{1} \mathrm{~J}=147.4 \mathrm{~Hz},{ }^{2} J\left(\mathrm{CH}_{2}, \mathrm{CH}_{2} \mathrm{CH}_{3}\right)=4.4 \mathrm{~Hz}\right), 119.7(\mathrm{Ph} \mathrm{C}-$ 2,6), $122.9\left(\mathrm{COCHCH},{ }^{1} J=165.9 \mathrm{~Hz},{ }^{2} J(\mathrm{C}, \mathrm{COCHCH})=3.6 \mathrm{~Hz}\right), 123.1\left(\mathrm{C}-4,{ }^{2} J(\mathrm{C} 4, \mathrm{H} 5)=7.3\right.$ $\mathrm{Hz}$ ), 128.0 (Ph C-4), 129.7 (Ph C-3,5), 131.3 (C-5, $\left.{ }^{1} J=188.1 \mathrm{~Hz}\right), 133.9\left(\mathrm{COCHCH},{ }^{1} J=164.7\right.$ $\left.\mathrm{Hz},{ }^{2} J(\mathrm{C}, \mathrm{COCHCH})=2.4 \mathrm{~Hz}\right), 138.9 \quad(\mathrm{Ph} \mathrm{C}-1), 148.3 \quad\left(\mathrm{C}-3,{ }^{2} J(\mathrm{C} 3, \mathrm{COCHCH})=3.2 \mathrm{~Hz}\right.$, $\left.{ }^{3} J(\mathrm{C} 3, \mathrm{COCHCH})=6.0 \quad \mathrm{~Hz}, \quad{ }^{3} J(\mathrm{C} 3, \mathrm{H} 5)=7.4 \quad \mathrm{~Hz}\right), \quad 166.6 \quad(\mathrm{COCHCH}), \quad 191.9 \quad\left(\mathrm{COCH}_{3}\right.$, $\left.{ }^{2} \mathrm{~J}\left(\mathrm{COCH}_{3}, \mathrm{CH}_{3}\right)=6.0 \mathrm{~Hz}\right)$; MS m/z (\%): $307\left([\mathrm{M}+\mathrm{Na}]^{+}, 100\right)$. Anal. Calcd for $\mathrm{C}_{16} \mathrm{H}_{16} \mathrm{~N}_{2} \mathrm{O}_{3} \cdot 0.3$ $\mathrm{H}_{2} \mathrm{O}: \mathrm{C}, 66.33$; H, 5.78; N, 9.67. Found: C, 66.37; H, 5.53; N, 9.28.

tert-Butyl (2E)-3-(4-acetyl-1-phenyl-1H-pyrazol-3-yl)acrylate (26). Brown liquid, yield 58\%, $91 \mathrm{mg}$; IR ( $\left.v_{\max }, \mathrm{cm}^{-1}\right): 3124\left(\mathrm{CH}_{\text {arom }}\right), 2978\left(\mathrm{CH}_{\text {aliph }}\right), 1700,1651(\mathrm{C}=\mathrm{O}), 754,690(\mathrm{CH}=\mathrm{CH}$ of monosubstituted benzene). ${ }^{1} \mathrm{H}$ NMR $\left(300 \mathrm{MHz}, \mathrm{CDCl}_{3}\right): \delta_{\mathrm{H}} 1.53\left(\mathrm{~s}, 9 \mathrm{H}, \mathrm{COO}\left(\mathrm{CH}_{3}\right)_{3}\right), 2.51(\mathrm{~s}$, $\left.3 \mathrm{H}, \mathrm{COCH}_{3}\right), 6.88\left(\mathrm{~d}, 1 \mathrm{H},{ }^{3} J_{\text {trans }}=16.11 \mathrm{~Hz}, \mathrm{COCH}\right), 7.37$ (m, 1H, Ph H-4), 7.49 (m, 2H, Ph H3,5), 7.73 (m, 2H, Ph H-2,6), 8.09 (d, 1H, $\left.{ }^{3} J_{\text {trans }}=16.11 \mathrm{~Hz}, \mathrm{COCHCH}\right), 8.35(\mathrm{~s}, 1 \mathrm{H}, \mathrm{H}-5) .{ }^{13} \mathrm{C}$

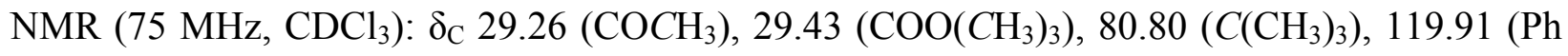
C-2,6), 123.32 (C-4), 125.11 (Ph C-4), 128.32 (CHCO), 129.93 (Ph C-3,5), 131.60 (C-5), 133.24 (CHCHCO), 139.23 (Ph C-1), 148.74 (C-3), 166.22 (CHCHCO), $192.21\left(\mathrm{COCH}_{3}\right)$; $\mathrm{MS} \mathrm{m} / z(\%)$ : $313\left([\mathrm{M}+\mathrm{H}]^{+}, 100\right)$. Anal. Calcd for $\mathrm{C}_{18} \mathrm{H}_{20} \mathrm{~N}_{2} \mathrm{O}_{3}: \mathrm{C}, 69.21 ; \mathrm{H}, 6.45 ; \mathrm{N}, 8.97$. Found: $\mathrm{C}, 69.61$; $\mathrm{H}, 6.49 ; \mathrm{N}, 8.94$.

1-\{1-Phenyl-3-[(E)-2-phenylethenyl]-1H-pyrazol-4-yl\}ethanone (27). White solid, yield 55\%, $79 \mathrm{mg}, \mathrm{mp} 95{ }^{\circ} \mathrm{C}$; IR $\left(v_{\max }, \mathrm{cm}^{-1}\right): 3127\left(\mathrm{CH}_{\text {arom }}\right), 1659(\mathrm{C}=\mathrm{O}), 756,687(\mathrm{CH}=\mathrm{CH}$ of monosubstituted benzenes). ${ }^{1} \mathrm{H}$ NMR $\left(500 \mathrm{MHz}, \mathrm{CDCl}_{3}\right): \delta_{\mathrm{H}} 2.53\left(\mathrm{~s}, 3 \mathrm{H}, \mathrm{CH}_{3}\right), 7.29(\mathrm{~m}, 1 \mathrm{H}$, CPh H-4), 7.37 (m, 2H, CPh H-3,5), 7.38 (m, 1H, NPh H-4), 7.51 (m, 2H, NPh H-3,5), 7.61 (m, $2 \mathrm{H}, \mathrm{CPh} \mathrm{H}-2,6), 7.69\left(\mathrm{~d}, 1 \mathrm{H},{ }^{3} J_{\text {trans }}=16.4 \mathrm{~Hz}, \mathrm{CHCHPh}\right), 7.77\left(\mathrm{~d},{ }^{3} J_{\text {trans }}=16.4 \mathrm{~Hz}, 1 \mathrm{H}\right.$, $\mathrm{CHCHPh}$ ), 7.78 (m, 2H, NPh H-2,6), 8.33 (s, 1H, H-5). $\left.{ }^{13} \mathrm{C} \mathrm{NMR} \mathrm{(125} \mathrm{MHz,} \mathrm{CDCl}_{3}\right): \delta_{\mathrm{C}} 29.0$ $\left(\mathrm{CH}_{3},{ }^{1} J=127.3 \mathrm{~Hz}\right), 118.4(\mathrm{PhCHCH}), 119.7(\mathrm{NPh} \mathrm{C}-2,6), 121.9\left(\mathrm{C}-4,{ }^{2} J(\mathrm{C} 4, \mathrm{H} 5)=7.5 \mathrm{~Hz}\right.$, $\left.{ }^{3} J(\mathrm{C} 4, \mathrm{CHCHPh})=1.2 \mathrm{~Hz}\right), 127.1(\mathrm{CPh} \mathrm{C}-2,6), 127.6$ (NPh C-4), 128.1 (CPh C-4), 128.6 (CPh C3,5), 129.6 (NPh C-3,5), 131.3 (C-5, ${ }^{1} J=186.6$ Hz), 133.1 (Ph-CH), 136.9 (CPh C-1), 139.2 $(\mathrm{NPh} \mathrm{C}-1), 151.0\left(\mathrm{C}-3,{ }^{2} J(\mathrm{C} 3, \mathrm{CHCHPh})=3.2 \mathrm{~Hz},{ }^{3} J(\mathrm{C} 3, \mathrm{CHCHPh})=6.4 \mathrm{~Hz},{ }^{3} J(\mathrm{C} 3, \mathrm{H} 5)=7.3 \mathrm{~Hz}\right)$, $192.4\left(\mathrm{CO},{ }^{2} J\left(\mathrm{CO}, \mathrm{CH}_{3}\right)=6.0 \mathrm{~Hz}\right) .{ }^{15} \mathrm{~N}$ NMR $\left(50 \mathrm{MHz}, \mathrm{CDCl}_{3}\right): \delta_{\mathrm{N}}-164.5(\mathrm{~N}-1),-85.6(\mathrm{~N}-2)$; MS $m / z(\%): 311\left([\mathrm{M}+\mathrm{Na}]^{+}\right.$, 100). Anal. Calcd for $\mathrm{C}_{19} \mathrm{H}_{16} \mathrm{~N}_{2} \mathrm{O}: \mathrm{C}, 79.14 ; \mathrm{H}, 5.59 ; \mathrm{N}, 9.72$. Found: C, 79.02; H, 5.63; N, 10.07 .

General procedure for the cyclization of 1-Phenyl-3-(phenylethynyl)-1H-pyrazole-4carbaldehyde (20) and corresponding ethanone (21)

A solution of 20 or $21(0.5 \mathrm{mmol})$ in dry ammonia and methanol $\left(\mathrm{NH}_{3} / \mathrm{MeOH} 2 \mathrm{M}, 8 \mathrm{ml}\right)$ was heated at $120{ }^{\circ} \mathrm{C}$ overnight in a steel reactor. The solvent was evaporated and the crude was 
purified by flash chromatography $\left(\mathrm{SiO}_{2}\right.$, eluent: ethyl acetate $/ n$-hexane, 1:4, v/v) to yield compounds $28,29$.

2,6-Diphenyl-2H-pyrazolo[4,3-c]pyridine (28). White solid, yield 89\%, $121 \mathrm{mg}, \mathrm{mp} 160{ }^{\circ} \mathrm{C}$; IR $\left(v_{\max }, \mathrm{cm}^{-1}\right): 3047\left(\mathrm{CH}_{\text {arom }}\right), 1622,1596,1506(\mathrm{C}=\mathrm{C}, \mathrm{C}-\mathrm{N}), 759,745,691(\mathrm{CH}=\mathrm{CH}$ of monosubstituted benzenes). ${ }^{1} \mathrm{H}$ NMR $\left(500 \mathrm{MHz}, \mathrm{CDCl}_{3}\right): \delta_{\mathrm{H}} 7.41(\mathrm{~m}, 1 \mathrm{H}, \mathrm{CPh} 4-\mathrm{H}), 7.47(\mathrm{~m}$, 1H, NPh 4-H), 7.50 (m, 2H, CPh 3,5-H), 7.57 (m, 2H, NPh 3,5-H), 7.93 (m, 2H, NPh 2,6-H), 8.03 (s, 1H, 7-H), 8.09 (m, 2H, CPh 2,6-H), 8.58 (s, 1H, 3-H), 9.33 (s, 1H, 4-H). ${ }^{13} \mathrm{C}$ NMR (125 $\left.\mathrm{MHz}, \mathrm{CDCl}_{3}\right): \delta_{\mathrm{C}} 107.0\left(\mathrm{C}-7,{ }^{1} J=164.0 \mathrm{~Hz},{ }^{4} J(\mathrm{C} 7, \mathrm{H} 4)=1.6 \mathrm{~Hz}\right), 119.7\left(\mathrm{C}-3,{ }^{2} J(\mathrm{C} 3 \mathrm{a}, \mathrm{H} 3)=8.1\right.$ $\left.\mathrm{Hz},{ }^{2} J(\mathrm{C} 3 \mathrm{a}, \mathrm{H} 4)=9.8 \mathrm{~Hz},{ }^{3} J(\mathrm{C} 3, \mathrm{H} 7)=4.2 \mathrm{~Hz}\right), 121.3(\mathrm{NPh} \mathrm{C}-2,6), 121.8\left(\mathrm{C}-3,{ }^{1} J=192.1 \mathrm{~Hz}\right)$, 127.0 (CPh C-2,6), 128.4 (CPh C-4), 128.7 (CPh C-3,5), 128.8 (NPh C-4), 129.8 (NPh C-3,5), 139.9 (CPh C-1), 140.0 (NPh C-1), 147.1 (C-4, $\left.{ }^{1} J=181.7 \mathrm{~Hz}\right), 151.3$ (C-6, $\left.{ }^{3} J(\mathrm{C} 6, \mathrm{H} 4)=12.4 \mathrm{~Hz}\right)$, $151.7\left(\mathrm{C}-7 \mathrm{a},{ }^{3} J(\mathrm{C} 7 \mathrm{a}, \mathrm{H} 3)=7.2 \mathrm{~Hz},{ }^{3} J(\mathrm{C} 7 \mathrm{a}, \mathrm{H} 4)=5.0 \mathrm{~Hz}\right) ;{ }^{15} \mathrm{~N}$ NMR $\left(50 \mathrm{MHz}, \mathrm{CDCl}_{3}\right): \delta_{\mathrm{N}}-145.9$ $(\mathrm{N}-2),-85.6(\mathrm{~N}-5), \mathrm{N}-1$ was not found; MS $m / z(\%): 272\left([\mathrm{M}+\mathrm{H}]^{+}, 100\right)$. Anal. Calcd for $\mathrm{C}_{18} \mathrm{H}_{13} \mathrm{~N}_{3} \bullet 0.4 \mathrm{H}_{2} \mathrm{O}$ : C, 77.62; H, 4.99; N, 15.09. Found: C, 77.48; H, 4.81; N, 15.46.

4-Methyl-2,6-diphenyl-2H-pyrazolo[4,3-c]pyridine (29). White solid, yield 91\%, $130 \mathrm{mg}, \mathrm{mp}$ $161{ }^{\circ} \mathrm{C}$; IR $\left(v_{\max }, \mathrm{cm}^{-1}\right): 3048\left(\mathrm{CH}_{\text {arom }}\right), 1611,1594(\mathrm{C}=\mathrm{C}, \mathrm{C}-\mathrm{N}), 772,745,696(\mathrm{CH}=\mathrm{CH}$ of monosubstituted benzenes). ${ }^{1} \mathrm{H}$ NMR $\left(500 \mathrm{MHz}, \mathrm{CDCl}_{3}\right): \delta_{\mathrm{H}} 2.89\left(\mathrm{~s}, 3 \mathrm{H}, \mathrm{CH}_{3}\right), 7.39(\mathrm{~m}, 1 \mathrm{H}$, CPh 4-H), 7.45 (m, 1H, NPh 4-H), 7.49 (m, 2H, CPh 3,5-H), 7.56 (m, 2H, NPh 3,5-H), 7.85 (s, 1H, 7-H), 7.92 (m, 2H, NPh 2,6-H), 8.08 (m, 2H, CPh 2,6-H), 8.53 (s, 1H, 3-H). ${ }^{13} \mathrm{C}$ NMR (125 $\left.\mathrm{MHz}, \mathrm{CDCl}_{3}\right): \delta_{\mathrm{C}} 23.3\left(\mathrm{CH}_{3},{ }^{1} J=127.3 \mathrm{~Hz},{ }^{4} J\left(\mathrm{CH}_{3}, \mathrm{H} 3\right)=1.0 \mathrm{~Hz}\right), 104.9\left(\mathrm{C}-7,{ }^{1} J=164.7 \mathrm{~Hz}\right)$, 119.8 (C-3a), 121.1 (NPh C-2,6), 121.6 (C-3, ${ }^{1} J=190.9$ Hz), 127.1 (CPh C-2,6), 128.6 (NPh C4), 128.2 (CPh C-4), 128.6 (CPh C-3,5), 129.7 (NPh C-3,5), 140.0 (NPh C-1), 140.1 (CPh C-1), $151.2(\mathrm{C}-6), 152.0\left(\mathrm{C}-7 \mathrm{a},{ }^{3} J(\mathrm{C} 7 \mathrm{a}, \mathrm{H} 3)=7.0 \mathrm{~Hz}\right), 155.8(\mathrm{C}-4) .{ }^{15} \mathrm{~N}$ NMR $\left(50 \mathrm{MHz}, \mathrm{CDCl}_{3}\right): \delta_{\mathrm{N}}$ $-148.2(\mathrm{~N}-2),-88.9(\mathrm{~N}-5),-75.8(\mathrm{~N}-1)$; MS m/z (\%): $286\left([\mathrm{M}+\mathrm{H}]^{+}, 100\right)$. Anal. Calcd for $\mathrm{C}_{19} \mathrm{H}_{15} \mathrm{~N}_{3} \bullet 0.6 \mathrm{H}_{2} \mathrm{O}$ : C, 77.06; H, 5.51; N, 14.19. Found: C, 76.77; H, 5.39; N, 14.39.

\section{References}

1. (a) Tsuji, J. Palladium Reagents and Catalysts-New Perspectives for the 21th Century; John Wiley and Sons Ltd: Chichester, 2004; pp 1-24. (b) Li, J. J.; Gribble G. W. Palladium in Heterocyclic Chemistry - a Guide for the Synthetic Chemist; Pergamon: Oxford, 2000; pp 134.

2. (a) Kotschy, A.; Timári, G. In Catalysis by Metal Complexes; James, B.; van Leeuwen, P. W. N. M. Eds.; Springer: Dordrecht, 2005; pp 97-194. (b) Cacchi, S.; Fabrizi, G. Chem. Rev. 2005, 105, 2873. (c) Banwell, M. G.; Goodwin, T. E.; Ng, S.; Smith, J. A.; Wong, D. J. Eur. J. Org. Chem. 2006, 3043.

3. Schnürch, M.; Flasik, R.; Khan, A. F.; Spina, M.; Mihovilovic, M. D.; Stanetty, P. Eur. J. Org. Chem. 2006, 3283. 
4. (a) Kleeman, A.; Engel, J.; Kutscher, B.; Reichert, D. Pharmaceutical Substances; 3rd Edn.; George Thieme: Stuttgart, New York, 1999; pp 1190-1787. (b) Dax, S. L. Antibacterial Chemotherapeutic Agents; Blackie Academic and Professional: London: Weinheim, New York, Melbourne, Madras, 1997; p 396. (c) Ha-Duong, N.-T.; Dijols, S.; Marques-Soares, C.; Minoletti, C.; Dansette, P. M.; Mansuy, D. J. Med. Chem. 2001, 44, 3622. (d) de Paulis, T.; Hemstapat, K.; Chen, Y.; Zhang, Y.; Saleh, S.; Alagille, D.; Baldwin, R. M.; Tamagnan, G. D.; Conn, P. J. J. Med. Chem. 2006, 49, 3332.

5. (a) Theodoridis, G. In Modern Crop Protection Compounds; Krämer, W.; U. Schirmer, U. Eds.; Wiley-VCH: Weinheim, 2007; Vol. 1, pp 153-186. (b) Shiga, Y.; Okada, I.; Ikeda, Y.; Takizawa, E.; Fukuchi, T. J. Pesticide Sci. 2003, 28, 313. (c) Lindell, S. D.; Moloney, B. A.; Hewitt, B. D.; Earnhaw, C. G.; Philip, P. J.; Dancer, J. E. Bioorg. Med. Chem. Lett. 1999, 9, 1985. (d) Vicentini, C. B.; Romagnoli, C.; Andreotti, E.; Mares, D. J. Agric. Food Chem. 2007, 55, 10331. (e) Fustero, S.; Román, R.; Sanz-Cervera, J. F.; Simón-Fuentes, A.; Bueno, J.; Villanova, S. J. Org. Chem. 2008, 73, 8545. (f) Dutra, G. A.; Hamper, B. C.; Mitschke, D. A.; Moedritzer, K.; Rogers, K.D. PCT Int. Appl., WO 8206, 962; Chem. Abstr. 1992, 117, 69859.

6. (a) Lu, Z.; Jiang, Q.; Zhu, W.; Xie, M.; Hou, Y.; Chen, X.; Wang, Z. Synth. Met. 2000, 111 112, 465. (b) Sano, T.; Fuji, Y.; Nishio, Y.; Hamada, Y. Jpn. J. Appl. Phys. 1995, 34, 3124. (c) Zhang, X. H.; Wu, S. K.; Gao, Z. Q.; Lee, C. S.; Lee, S. T.; Kuong, H. L. Thin Solid Films 2000, 371, 40.

7. (a) Costa, D. C.; Ortí, E.; Bolink, H. J.; Graber, S.; Housecroft, C. E.; Constable, E. C. J. Am. Chem. Soc. 2010, 132, 5978. (b) Martínez, A. P.; Fabra, M. J.; García, M. P.; Lahoz, F. J.; Oro, L. A.; Teat, S. J. Inorg. Chim. Acta 2005, 358, 1635. (c) Churruca, F.; SanMartin, R.; Tellitu, I.; Domínguez, E. Synlett 2005, 20, 3116.

8. Arbačiauskienė E.; Vilkauskaitė G.; Eller G.; Holzer, W.; Šačkus A. Tetrahedron 2009, 65, 7817.

9. (a) Guillou, S.; Nesmes, O.; Ermolenko, M. S.; Janin, Y. L. Tetrahedron 2009, 65, 3529. (b) Stauffer, S. R.; Huang, Y.; Coletta, C. J.; Tedesco, R.; Katzenellenbogen, J. A. Bioorg. Med. Chem. 2001, 9, 141. (c) Guillou, S.; Bonhomme, F. J.; Janin, Y. L. Tetrahedron 2009, 65, 2660; (d) Majo, V. J.; Prabhakaran, J.; Mann, J. J.; Kumar, J. S. D. Adv. Synth. Cat. 2003, 345, 620. (d) Raboisson, P.; Schultz, D.; Muller, C.; Reimund, J.-M.; Pinna. G.; Mathieu, R.; Bernard, P.; Do, Q.-T.; DesJarlais, R. L.; Justiano, H.; Lugnier, C.; Bourguignon, J.-J. Eur. J. Med. Chem. 2008, 43, 816; (e) Wang, X.; Tan, J.; Grozinger, K. Tetrahedron Lett. 2000, 41, 4713. (f) Heinisch, G.; Holzer, W.; Obala, C. Monatsh. Chem. 1988, 119, 253.

10. (a) Pinkerton, A. B.; Huang, D.; Cube, R. V.; Hutchinson, J. H.; Struthers, M.; Ayala, J. M.; Vicario, P. P.; Patel, S. R.; Wisniewski, T.; DeMartino, J. A.; Vernier, J.-M. Bioorg. Med. Chem. Lett. 2007, 17, 807. (b) Zhang, J.; Zhang, Y.; Schnatter, W. F. K.; Herndon, J. W. Organometallics 2006, 25, 1279. (c) Gorja, D. R.; Batchu, V. R.; Ettam, A.; Pal, M. Beilstein J. Org. Chem. 2009, 5, 1. (d) Tretyakov, E. V.; Knight, D. W.; Vasilevsky, S. F. J. Chem. 
Soc., Perkin Trans. 1 1999, 3713. (e) Zoppellaro, G.; Baumgarten, M. Eur. J. Org. Chem. 2005, 2888.

11. (a) Dow, R. D.; Carpino, P. A.; Hadcock, J. R.; Black, S. C.; Iredale, P. A.; DaSilva-Jardine, P.; Schneider, S. R.; Paight, E. S.; Griffith, D. A.; Scott, D. O.; O’Connor, R. E.; Nduaka, C. ффI. J. Med. Chem. 2009, 52, 2652. (b) Jeon, S. L.; Choi, J. H.; Kim, B. T.; Jeong, I. H. J. Fluorine Chem. 2007, 128, 1191.

12. (a) Dvorak, C. A.; Rudolph, D. A.; Ma, S.; Carruthers, N. I. J. Org. Chem. 2005, 70, 4188.

(b) Raimundo, B. C.; Oslob, J. D.; Braisted, A. C.; Hyde, J.; McDowell, R. S.; Randal, M.; Waal, N. D.; Wilkinson, J.; Yu, C. H.; Arkin M. R. J. Med. Chem. 2004, 47, 3111. (c) Dirat, O.; Clipson, A.; Elliott, J. M.; Garrett, S.; Jones, A. B.; Reader, M.; Shaw, D. Tetrahedron Lett. 2006, 47, 1729. (d) Dragovich, P. S.; Bertolini, T. M.; Ayida, B. K.; Li, L.-S.; Murphy, D. E.; Ruebsam, F.; Sun, Z.; Zhou, Y. Tetrahedron 2007, 63, 1154.

13. Zhang, T.; Gao, X.; Wood, H. B. Tetrahedron Lett. 2011, 52, 311.

14. Chandra, A.; Singh, B.; Upadhyay, S.; Singh, R. M. Tetrahedron 2008, 64, 11680.

15. (a) Čikotienė I.; Bukšnaitienè, R.; Sazinas, R. Tetrahedron 2011, 67, 706. (b) Čikotienė, I.; Kairys, V.; Bukšnaitienè, R.; Morkūnas, M.; Rudys, S.; Brukštus, A.; Fernandes, M. X. Tetrahedron 2009, 65, 5752.

16. Arcadi, A.; Attanasi, O. A.; Guidi, B.; Rossi, E.; Santeusanio, S. Eur. J. Org. Chem. 1999, 3117.

17. Portela-Cubillo, F.; Lymer, J.; Scanlan, E. M.; Scott, J. C.; Walton, J. C. Tetrahedron 2008, $64,11908$.

18. O’Brien, D. F.; Gates, J. W., Jr. J. Org. Chem. 1966, 31, 1538.

19. (a) Yu, M.; Tsuyoshi, M.; Hiroshi, I.; Hiroyuki, O.; Hiroyuki, K. PCT Int. Appl., WO 2002076959; Chem. Abstr. 2002, 137, 279197. (b) Yu, M.; Nobuyuki, T.; Tsuyoshi, M.; Hiroyuki, O.; Hiroyuki, K. PCT Int. Appl., WO 2004024705; Chem. Abstr. 2004, 140, 287404.

20. Felding, J.; Kristensen, J.; Bjerregaard, T.; Sander, L.; Vedsø, P.; Begtrup, M. J. Org. Chem. 1999, 64, 4196.

21. (a) Balle, T.; Vedsø, P.; Begtrup, M. J. Org. Chem. 1999, 64, 5366. (b) Stensbøl, T. B.; Uhlmann, P.; Morel, S.; Eriksen, B. L.; Felding, J.; Kromann, H.; Hermit, M. B.; Greenwood, J. R.; Braüner-Osborne, H.; Madsen, U.; Junager, F.; Krogsggard-Larsen, P.; Begtrup, M.; Vedsø, P. J. Med. Chem. 2002, 45, 19. (c) Vedsø, P.; Begtrup, M. J. Org. Chem. 1995, 60, 4995. (d) Arbačiauskienė E.; Jankauskas V.; Kazlauskas K.; Miasojedovas A.; Juršènas S.; Holzer W.; Getautis V.; Šačkus A. Dyes and Pigments 2010, 85, 79. (e) Arbačiauskienė E.; Kazlauskas K.; Miasojedovas A.; Juršènas S.; Jankauskas V.; Holzer W.; Getautis V.; Šačkus A. Synth. Metals 2010, 160, 490.

22. (a) Vera-DiVaio, M. A. F.; Freitas, C. C. A.; Castro, H. C.; de Albuquerque, S.; Cabral, L. M.; Rodrigues, C. R.; Albuquerque, M. G.; Martins, R. C. A.; Henriques, M. G. M. O. Bioorg. Med. Chem. 2009, 17, 295. (b) Luo, Y.; Zhong, P.; Zhang, X. H.; Lin, Q. L.; Chen, Y. N. Chinese Chem. Lett. 2008, 19, 383. 
23. Dai, H.; Yu, H.-B.; Liu, J.-B.; Li, Y.-Q.; Qui, X.; Zhang, X.; Qin, Z.-F.; Wang, T.-T.; Fang, J.-X. Arkivoc 2009, (vii), 126.

24. Fletcher, S.; Gunning, P. T. Tetrahedron Lett. 2008, 49, 4817.

25. (a) Jutland, A.; Mosleh, A. Organometallics 1995, 14, 1810; (b) Lutz, C.; Bleicher, K. H. Tetrahedron Lett. 2002, 43, 2211.

26. (a) Bernard, M.; Hulley, E.; Molenda, H.; Stochla, K.; Wrzeciono, U. Pharmazie 1986, 41, 560. (b) Kira, M. A.; Abdel-Rahman, M. O.; Gadalla, K. Z. Tetrahedron Lett. 1969, 2, 109. (c) Prakash, O.; Pannu, K.; Kumar, A. Molecules 2006, 11, 43. (d) Kumar, R. S.; Karthikeyan, K.; Perumal. P. T. Can. J. Chem. 2008, 86, 720. (e) De Luca, L.; Giacomelli, G.; Masala, S.; Porcheddu, A. Synlett 2004, 13, 2299.

27. For example: (a) Youssef, A. M.; White, M. S.; Villanueva, E. B.; El-Ashmawy, L. M.; Klegeris, A. Bioorg. Med. Chem. 2010, 18, 2019. (b) Bandgar, B. P.; Gawande, S. S.; Bodade, R. G.; Gawande, N. M.; Khobragade, C. N. Bioorg. Med. Chem. 2009, 17, 8168. (c) Rathelot, P.; Azas, N.; El-Kashef, H.; Delmas, F.; Di Giorgio, C.; Timon-David, P.; Maldonado, J.; Vanelle, P. Eur. J. Med. Chem. 2002, 37, 671.

28. Vovk, M. V.; Mel'nichenko, N. V.; Chornous, V. A.; Bratenko, M. K. Chem. Het. Comp. 2002, 38, 1096.

29. (a) Adembri, G.; Celli, A. M.; Scotton, M. J. Het. Chem. 1998, 25, 249. (b) Huisgen, R.; Gotthardt, H.; Grashey, R. US Pat. 3254093, 1966; Chem. Abstr. 1966, 65, 38548. 